\title{
Simulations of ARGO profilers and of surface floating objects: applications in MFSTEP
}

\author{
C. Pizzigalli and V. Rupolo \\ Climate Department, ENEA, Via Anguillarese 30100060 Rome, Italy \\ Received: 5 October 2006 - Published in Ocean Sci. Discuss.: 20 October 2006 \\ Revised: 19 March 2007 - Accepted: 5 April 2007 - Published: 4 May 2007
}

\begin{abstract}
In this work we describe part of the activities performed in the MFSTEP project by means of numerical simulations of ARGO profilers and surface floating objects. Simulations of ARGO floats were used to define the optimal time cycling in order to maximize independent observations of vertical profiles of temperature and salinity and to minimize the error on the estimate of the velocity at the parking depth of the profilers. Instead, the Mediterranean Forecasting System archive of Eulerian velocity field from 2000 to 2004 was used to build a related surface Lagrangian archive, systematically integrating numerical particles released and constrained to drift at surface. Here we use such Lagrangian archive to study the interannual variability of the surface Lagrangian transport in two key areas of the Western Mediterranean, also introducing an exponential decay in the particles concentration.
\end{abstract}

\section{Introduction}

The Eulerian description of the flow is obviously of primary importance for the knowledge of the dynamics of the sea, but often critical questions concerning the path of the water masses are much more easily accessible from the Lagrangian picture of the motion. Moreover, Lagrangian experimental devices provide economical observations over extended areas for long time periods, even during extreme weather conditions, and play by now a major role in the global ocean observing system. These simple observations have motivated, as one important enrichment of the general architecture of the previous Mediterranean Forecasting System Pilot Project (MFSPP), the introduction of the Lagrangian framework, both in the modelling and observing part of MFSTEP.

Correspondence to: V. Rupolo

(rupolo@ casaccia.enea.it)
During the project, starting from June 2004, about twenty autonomous drifting ARGO profilers were deployed in the Mediterranean Sea in the context of the MedARGO project that is fully described in the papers of Poulain (2005) and Poulain et al. (2006). ARGO floats freely drift at a prescribed depth for a given time interval and then they resurface and transmit to the satellite ARGOS system position data and vertical Temperature and Salinity (TS) profiles, before starting a new cycle. In the global oceans, data from autonomous drifting profilers are collected in the framework of the ARGO international project (http://www.argo.net) as a part of the global ocean observing system whose main aim is to collect data to detect and study climate changes. ARGO vertical profiles are collected typically at 10 days interval in the upper $2000 \mathrm{~m}$. Contrastingly, MedARGO data are collected to provide Near Real Time (NRT) TS data to be assimilated, together with the Temperature profiles from the Volunteer Observing Ships (Manzella et al., 2003), in an operational forecasting model of a basin of reduced size and characterized by a rather complex bathymetric structure. Other than TS vertical profiles, autonomous drifters profilers provide also information about the velocities at the parking depth that may also be assimilated in a General Circulation Model (Molcard et al., 2005). However, this estimate, being obtained directly from the resurfacing positions of the ARGO profilers, do not consider residual motions at intermediate depth and it is affected by the intrinsic errors given by the neglected horizontal displacements during the vertical motion of the profiler. Consequently, designing the overall MFSTEP architecture it was foreseen a research activity devoted to define, by means of numerical simulations, the 'best' cycling characteristics for the MedARGO floats in order to maximize independent observations of vertical profiles of TS and to study the error on the estimate of intermediate velocity, its dependence on the time characteristics of the profiler cycle and, possibly, on the geographic area of release. The technique adopted for these purposes is described in detail in this paper, while

Published by Copernicus GmbH on behalf of the European Geosciences Union. 
in Poulain et al. (2006) only the results are presented. During MFSTEP, numerical simulations of the ARGO profilers movement were also used to quantify the impact of assimilating in the forecasting model vertical TS profiles (Griffa et al., 2006; Raicich 2006) and positions (Taillandier and Griffa, 2006) and to design an array of profiling floats for the estimation of the 3-D thermohaline fields (Guinehut et al., 2002).

Eulerian velocity fields from MFSPP were also used to compute numerical trajectories to study in the Mediterranean the variability of the surface dispersion, since a better description of its phenomenology is an important oceanographic issue both for operational and scientific purposes. For operational purposes, the knowledge of surface dispersal properties is useful in case of pollutant releases at sea (e.g. oil spill), for the assessment of biological quantities such as larvae spreading and in the search and rescue activities, to make a first guess at the most probable direction of drifting. For scientific purposes, information about the Lagrangian dispersion variability at sea surface is an appropriate tool to study the heat and salt budgets in an evaporative basin like the Mediterranean Sea, where the hydrological properties of the surface layers often considerably differ from those of the sub-surface layers and where, due to the presence of geomorphic constraints, the variability of the surface flow may influence the basin-wide thermohaline circulation through modification of the heat and salinity contents (Astraldi et al., 1992). Technically, using MFSPP Eulerian hindcast velocity fields from the 2000 to 2004 , we have built a huge Lagrangian archive systematically integrating particles released, and constrained to drift, at surface. This "Lagranian atlas", obtained from velocity fields in which the effects of the variability of the surface forcing is fully represented, was already used to study, following a statistical approach, the seasonal variability of surface dispersion at basin scale (Pizzigalli et al., 2007). In this work we present a further example of exploitation of this Lagrangian data set investigating the interannual variability of the "Lagrangian transport" in two key areas of the Mediterranean Sea considering also the case of tracers characterized by a concentration decreasing with an exponential decay.

Numerical simulation of both ARGO profilers and surface floating objects were done using the basin wide Eulerian velocity fields from the Med831 model (MOM 1/8 $\times 1 / 8^{\circ} \times 31$ levels, model details are described in Korres at al., 2000, and Demirov et al., 2003). Obviously, all the results presented in this study depend on the performance of the Eulerian model. The scarce space and time resolution does not allows for a proper representation of the high-frequency sub inertial and mesoscale motions and among other model deficiencies (e.g. unsophisticated representation of sub-grid scale effects) likely causing discrepancies between real and modelled floating objects, one can mention the lack of a parameterization representing the wave effects. Finally, the space resolution (about $0.5^{\circ}$ ) of the ECMWF (European Cen- tre for Medium-Range Weather Forecasts) analysis used to force the MFSPP OGCM may not be sufficient to properly represent the wind-induced surface dynamics especially in a basin in which the neighbouring lands are characterized by complex orography. However, the MFSPP Eulerian velocity fields used in this study, obtained from a model which assimilates in-situ real data and is forced by analyzed wind fields, represent the best basin scale description one can get for the variability of the circulation patterns from the model grid size scale $h$ to the Mediterranean sub-basins scale, whose typical dimension is of the order of $300-500 \mathrm{~km}$, i.e. for times $t$ : $\frac{h}{V} \approx 1$ day $<t<\frac{300-500 \mathrm{~km}}{\mathrm{~V}} \approx 30$ days, where $V \approx 10 \mathrm{~cm} / \mathrm{s}$ is the typical scale of velocity.

Comparing numerical and real Lagrangian trajectories is perhaps one of the most stringent model validation procedure. When dealing with ARGO simulations we will compare some statistical properties of the cycles of numerical and real MedARGO profilers, which may be considered as an indirect validation procedure to check the ability of the OGCM to represent subsurface flow. In Pizzigalli et al. (2007) we check the results obtained from a seasonal statistics of numerical surface dispersion with experimental data from the Mediterranean Surface Drifter Database (Poulain et al., 2004) showing that the MFSPP OGCM reasonably well represent the variability of dispersion in such time scales (from few days to few weeks). The results presented here concerning the interannual variability of the surface Lagrangian transport in the Corsica and Sardinia Channels may hardly be checked against real data. However, this "intra-basin" transport occurs on space and time scales well resolved by the model and it is mainly driven by atmospheric features whose relatively low space and time variability is well represented in the ECMWF wind fields. We consequently believe that the results presented here, possibly compared with other numerical studies and when possible with real Lagrangian data, may be still be useful to study and assess the variability of the surface circulation in the Mediterranean Sea.

This work is composed by two different and separate parts. In Sect. 2 we describe in detail the use of the numerical simulations of the ARGO profilers for the definition of the optimal sampling strategy and we discuss the statistics of the errors on the determination of the sub-surface velocity from ARGO data. In Sect. 3 we show some results concerning the interannual variability of the Lagrangian transport in the Corsica and Sardinia Channels taking also into account the dilution rate of the tracer due, e.g., to the evaporation of the given pollutant or to the larvae mortality. Finally, Sect. 4 summarizes the results and discusses some future prospects, notably in terms of development and improvement of the use of Lagrangian numerical tools in operational projects. 


\section{Numerical ARGO profilers}

\subsection{Numerical algorithm}

The motion of the profiler is simulated through an off-line algorithm obtained modifying the original code ARIANE (http://www.ifremer.fr/lpo/blanke/ARIANE/index. html; Blanke and Raynaud, 1997). During a profiler cycle (see Fig. 1) the simulated profiler downwells to a specified depth $Z_{\text {drift }}$, where it freely drifts for a given time $T_{\text {drift }}$, then further downwells to a second specified depth $Z_{\text {down }}$ to immediately upwells to the surface, where it stays for a given time $T_{\text {surf. }}$. During the vertical displacements the floats are subjected to the horizontal movements induced by the vertical velocity shear. For the real profilers of the MedARGO program the "parking" depth $Z_{\text {drift }}$ was chosen to be fixed at $350 \mathrm{~m}$, that approximately corresponds to the "mean core depth" of the Levantine Intermediate Water, while the deepest depth is fixed at $700 \mathrm{~m}$ (Poulain et al., 2006). For some floats and every ten cycles the floats downwells to a depth $Z_{\text {down }}=2000 \mathrm{~m}$. In each numerical experiment the parking and the deepest depths were fixed at $350 \mathrm{~m}$ and at $700 \mathrm{~m}$, (for all the cycles), while, during the vertical displacements, the "numerical profilers" move with the constant experimental-like downward and upward velocities $w=-5 \mathrm{~cm} / \mathrm{s}$ and $w=10 \mathrm{~cm} / \mathrm{s}$, respectively.

In each cycle the profiler stays at surface for a time $T_{\text {surf }}=T_{\text {surf } 1}+T_{\text {surf } 2}+T_{\text {surf3 }}$ where $T_{\text {surf1 }}=T_{\text {surf } 2} \approx 15$ min represent, respectively, the time intervals between the last surface positioning and the sinking and between the resurfacing and the first satellite positioning of the float and $T_{\text {surf } 3} \approx 6 \mathrm{~h}$ is the time spent at surface to transmit data. The time interval $T_{\text {tot }}$ between the last float positioning in the surface and the first positioning after the re-surfacing is given (see Fig. 1) by:

$T_{\text {tot }}=T_{\text {surf1 }}+T_{\text {down } 1}+T_{\text {drift }}+T_{\text {down } 2}+T_{\text {upw }}+T_{\text {surf2 }}$,

where $T_{\text {down } 1}=T_{\text {down2 }}=\frac{350 \mathrm{~m}}{5 \mathrm{~cm} / \mathrm{s}} \approx 2 \mathrm{~h}$ is the time necessary for the first (from surface to $350 \mathrm{~m}$ ) and the second (from 350 to $700 \mathrm{~m}$ ) upwelling and $T_{\text {upw }}=\frac{700 \mathrm{~m}}{10 \mathrm{~cm} / \mathrm{s}} \approx 2 \mathrm{~h}$ is the time necessary for the upwelling of the profiler to the surface. During the horizontal and vertical movements the profiler trajectory is sampled every $3 \mathrm{~h}$ and every $18 \mathrm{~s}$, respectively. The code is flexible and the user can easily change the depth and the time characteristics of the cycles.

\subsection{Numerical experiments and cycles statistics}

We have performed 6 experiments varying the time $T_{\text {drift }}$ from 3 to 30 days. In each experiment 40136 "numerical profilers" are uniformly released at surface (with an approximate density of 4 particles $/ 100 \mathrm{~km}^{2}$ ) only where the bottom sea is deeper than $700 \mathrm{~m}$, with the exceptions of the shallow North Aegean, Adriatic Sea and Sicily Channel (see Fig. 2). After the release each numerical profiler sinks to the equilibrium depth of $350 \mathrm{~m}$ and starts its cycles. The integration is

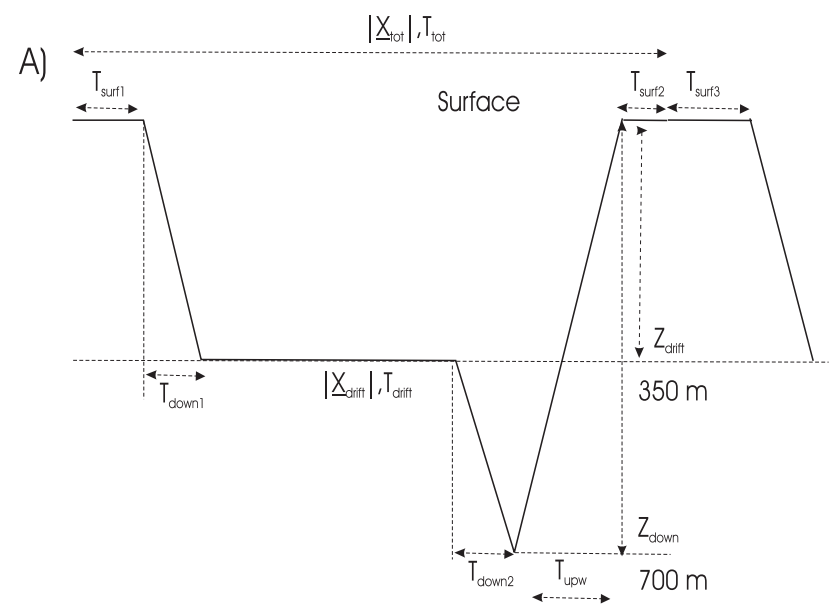

B)

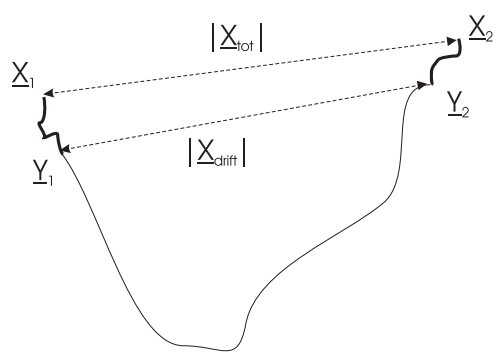

Fig. 1. Schematic and notations used in the text for a typical profiler cycle.

performed for 364 days using the 3 days mean eulerian hindcast velocity field of the year 2000 (52 weeks) obtained with the Med831 model (MOM $1 / 8^{\circ} \times 1 / 8^{\circ} \times 31$, see Korres at al., 2000, and Demirov et al., 2003).

When a real ARGO float crashes on the bottom it simply stays at the floor sea for the described time and then it resurfaces. In our simulations, if the numerical profiler reaches the bottom it immediately resurfaces to start a new cycle and, to avoid spurious results, we consider in our statistics only cycles in which the numerical floats do not reach the bottom. Consequently we have a statistics based on a number of cycles $N_{\text {cycles }} \neq N_{t c}=N_{\text {part }} \cdot \frac{T_{\text {INT }}}{T_{\text {tot }}}$, where $N_{\text {part }}$ is the number of profilers and $T_{\mathrm{INT}}=364$. For each experiment $N_{\text {cycles }}$ is always very large (see Table 1) and the ratio $\frac{N_{\text {cycles }}}{N_{t c}}$ is about 0.66 for EXP1 and about 0.75 for the five experiments EXP2EXP6. From 2004 to the end of 2006 are available $1791 \mathrm{cy}-$ cles of MedARGO floats with 4.5 days $<T_{\mathrm{Tot}}<5.5$ days (for real MedARGO floats $T_{\text {drift }}=5$ days), that were downloaded from the ARGO Data Center. The ARGO netcdf files contain for each float cycle a parameter indicating, when known, if the float has touched the ground. Unfortunately for more than $80 \%$ of cycles this parameter results to be "unknown" making impossible a direct comparison of the percentage of cycles touching the sea floor between numerical and real data. 


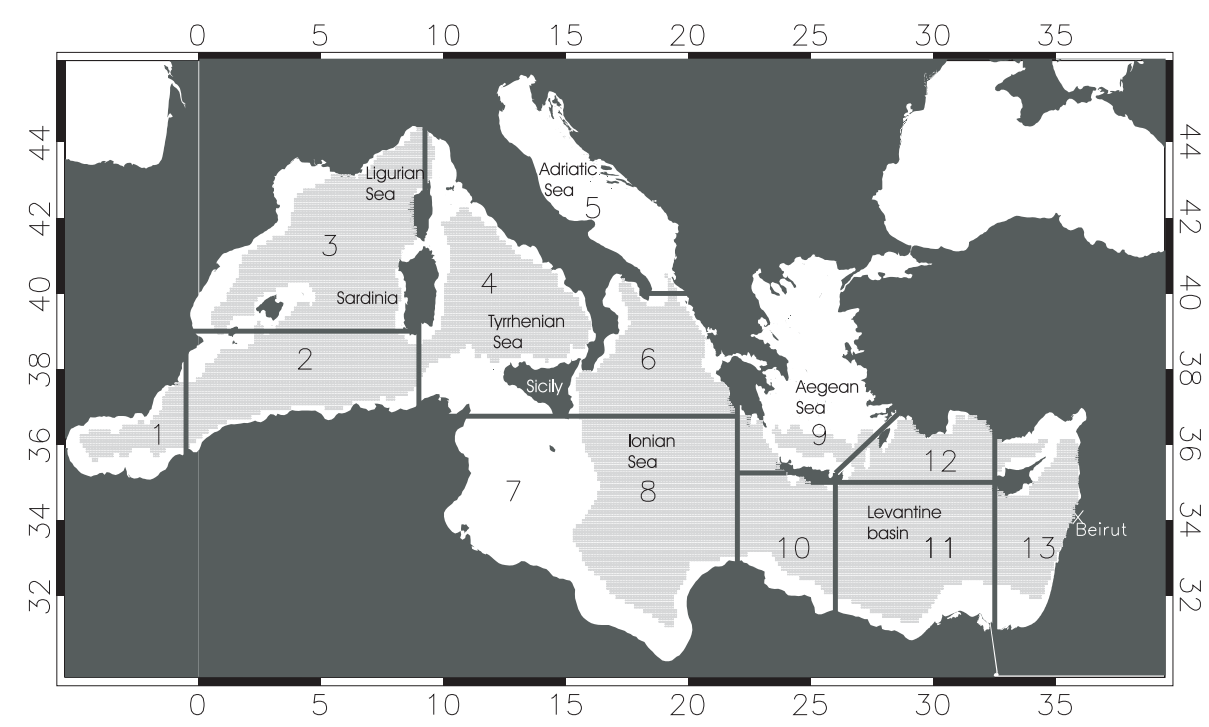

Fig. 2. Numerical profilers are uniformly released at surface in the grey light area. Numbers indicate the different regions discussed in the text.

Table 1. $T_{\text {drift }}$, total number of cycles and number of cycles characterized by having $\left|X_{\text {tot }}\right|$ greater than 10 and $20 \mathrm{~km}$ for MedARGO data and the six numerical experiments. In each experiment were integrated 40136 numerical profilers uniformly released at surface.

\begin{tabular}{ccccc}
\hline & $T_{\text {drift }}($ days $)$ & $N_{\text {cycles }}$ & $\left|X_{\text {Tot }}\right|>10$ & $\left|X_{\text {Tot }}\right|>20$ \\
\hline MedARGO & 5 & 1791 & $1190(66 \%)$ & $581(32 \%)$ \\
EXP1 & 3 & 3207232 & $1913600(60 \%)$ & $814419(25 \%)$ \\
EXP2 & 6 & 1809631 & $1323463(73 \%)$ & $881675(49 \%)$ \\
EXP3 & 9 & 1229731 & $1006347(82 \%)$ & $778891(63 \%)$ \\
EXP4 & 12 & 917250 & $798536(87 \%)$ & $665921(73 \%)$ \\
EXP5 & 15 & 739580 & $668086(90 \%)$ & $580922(79 \%)$ \\
EXP6 & 30 & 365745 & $350905(96 \%)$ & $329546(90 \%)$ \\
\hline
\end{tabular}

However, we may obtain a rough estimate of the number of cycles touching the sea floor considering the points that lie in the band defined by the two lines $Z_{\mathrm{Max}}=Z_{\mathrm{Bot}} \pm|\mathrm{Err}|$, where $Z_{\mathrm{Max}}$ is the deepest depth reached by the floats, $Z_{\mathrm{Bot}}$ is the bottom depth at the location of the profile and the error Err may be due or to the indeterminacy of the position or to the discretised topography data set. Considering values of Err of 50 and $100 \mathrm{~m}$, we have that about the $6 \%$ and $9 \%$ of the points of the scatter plot shown in Fig. 3a lie in such bands, which indicates that the numerical results overestimate the number of cycles in which the float touches the sea floor, even if it has to be stressed that many numerical profilers are released near the isobath of $700 \mathrm{~m}\left(Z_{\text {down }}=700 \mathrm{~m}\right)$.

In the two last columns of Table 1, we show the number and percentages of cycles in which $\left|X_{\text {Tot }}\right|$ is greater than 10 and $20 \mathrm{~km}$, which may be considered as a proxy of number of consecutive independent vertical profiles of TS, since the Rossby deformation radius that in the Mediterranean Sea is of the order of $\mathrm{O}(5-15) \mathrm{km}$. The percentage of consecutive and independent observations tends to saturate to $100 \%$ only for very big $T_{\text {drift }}$. For $T_{\text {drift }}=6$ days (EXP2) we have that about the $73 \%$ and $49 \%$ of cycles have $\left|X_{\text {Tot }}\right|>10$ and $20 \mathrm{~km}$, respectively. The number of cycles with $\left|X_{\text {Tot }}\right|>10 \mathrm{~km}$ is greater for EXP1 while the number of cycles with $\left|X_{\text {Tot }}\right|>20 \mathrm{~km}$, slightly larger for EXP2, is almost similar for EXP1, EXP2 and EXP3. These results was used to define the time cycling characteristics of the real MedARGO floats, and it is encouraging to note that the numerical results are in general agreement with real data since the percentage of MedARGO cycles with $\left|X_{\text {Tot }}\right|>10$ and $20 \mathrm{~km}$ lies between results from EXP1 and EXP2 (see also Fig. 3b). 

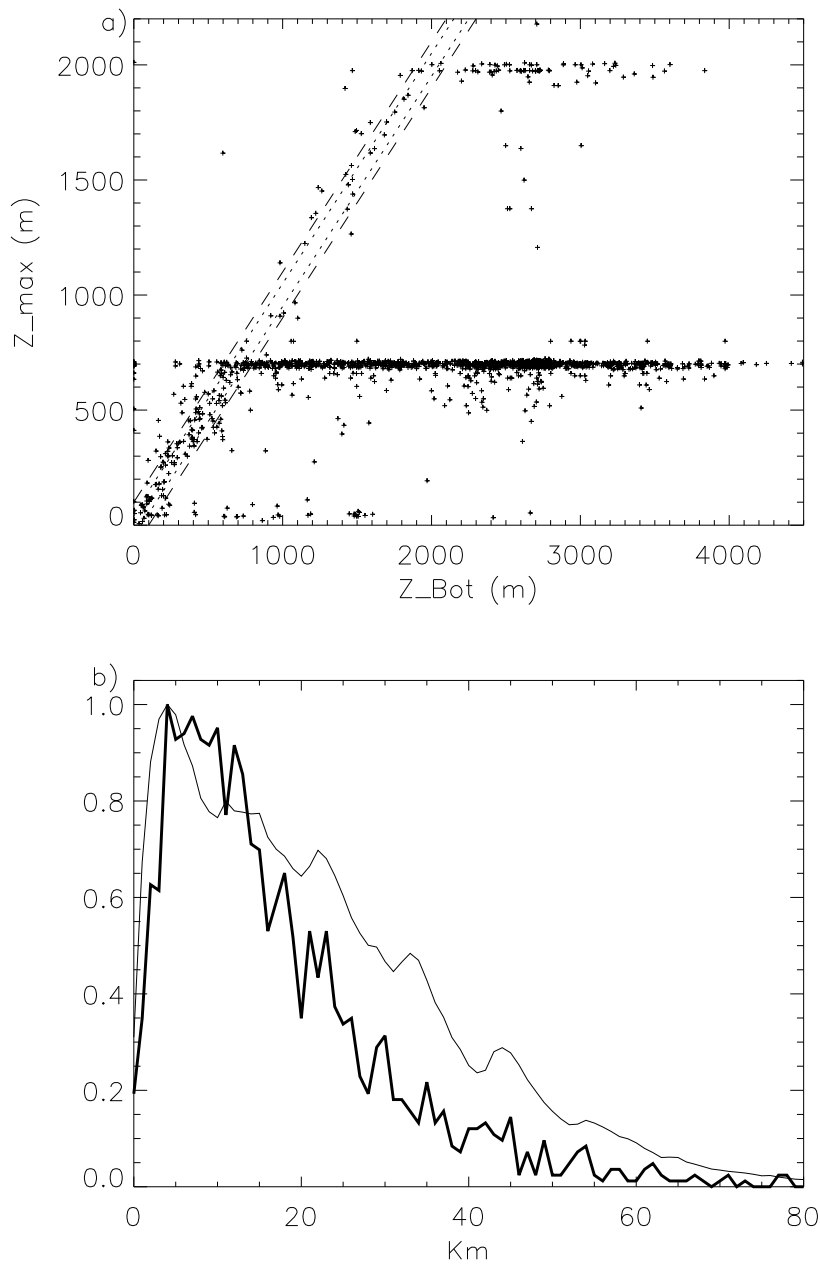

Fig. 3. Panel (a) scatter plot of maximum depth of experimental Medargo cycles (1791) vs. bottom depth at the resurfacing location. Bottom depth is retrieved from the Digital Bathymetric Data Base at $1^{\prime}$ resolution and was kindly provided by $\mathrm{M}$. Tonani (INGV). Dotted and dashed lines are defined by $Z_{\mathrm{Max}}=Z_{\mathrm{BOT}} \pm \mid$ Err $\mid$ with Err $=50 \mathrm{~m}$ and $\mathrm{Err}=100 \mathrm{~m}$, respectively. Panel (b) Histogram of distance between two subsequent resurfacing locations for EXP2 and MedARGO real data (thin and thick line, respectively). We consider only real cycles which passed all automatic quality control test and separated by a time interval 4.5 days $<T_{\text {Tot }}<5.5$ days.

\subsection{Sub-surface velocities}

The estimate of the intermediate currents by means of ARGO floats is given by

$U=\frac{\left|\underline{X}_{\text {Tot }}\right|}{T_{\text {tot }}}=\frac{\left|\underline{X}_{2}-\underline{X}_{1}\right|}{T_{\text {tot }}}$

where $\underline{X}_{i}$ are the surface positions before and after the deep cycle of the float and $T_{\text {tot }}$ is the time interval between these two subsequent, satellites located, surface points (see Fig. 1). This estimate (Eq. 1) is inadequate when $T_{\text {drift }}$ is greater or comparable to the intermediate velocity Lagrangian corre-
Table 2. Percentage of cycles characterized by a ratio $\frac{\left|\underline{X}_{\text {drift }}\right|}{D}<L$.

\begin{tabular}{ccccc}
\hline & $L=0.9$ & $L=0.8$ & $L=0.7$ & $L=0.6$ \\
\hline EXP1 & $2 \%$ & $1 \%$ & $<1 \%$ & $<1 \%$ \\
EXP2 & $6 \%$ & $3 \%$ & $1 \%$ & $1 \%$ \\
EXP3 & $13 \%$ & $6 \%$ & $2 \%$ & $2 \%$ \\
EXP4 & $21 \%$ & $10 \%$ & $4 \%$ & $3 \%$ \\
EXP5 & $29 \%$ & $15 \%$ & $6 \%$ & $4 \%$ \\
EXP6 & $57 \%$ & $37 \%$ & $18 \%$ & $13 \%$ \\
\hline
\end{tabular}

lation time $T_{L}$ since in this case, due to the presence of eddies and meanders, the length of the piece of trajectory $D=\int_{T_{j}}^{T_{j}+T_{\text {drift }}} d \underline{Y}$ covered by the profiler in the time $T_{\text {drift }}$ is greater than $\left|\underline{X}_{\text {drift }}\right|=\left|\underline{Y}_{2}-\underline{Y}_{1}\right|$, where $\underline{Y}_{i}$ represent coordinates of the first and last point of the trajectory at the parking depth $Z_{\text {drift. }}$ A second source of inaccuracy is given by the fact that the definition (1) does not take into account the horizontal displacements occurring at the surface, first and before the satellite locations, and during the vertical motions of the profiler $\left(\left|\underline{X}_{\text {Tot }}\right| \neq\left|\underline{X}_{\text {drift }}\right|\right.$ in the notation of Fig. 1).

We have then computead, for each cycle of the six experiments EXP1-EXP6, $\left|\underline{X}_{\text {drift }}\right|$ and the distance $D$ covered by the numerical profilers, approximated as a broken line of segments of time length equal to the time interval of the Lagrangian integration $(3 \mathrm{~h}$ ). From Table 2, where we report for each experiment the percentage of cycles in which the ratio $\frac{\left|X_{\text {drift }}\right|}{D}$ is smaller than a threshold value $L$, it is possible to see that a significant number of cycle $(>5 \%)$ have an important difference $(L<0.8)$ for $T_{\text {drift }} \geq 9$ days, time scale that may be considered as a crude estimate of the order of magnitude of $T_{L}$ from the numerical Lagrangian velocity time series at $350 \mathrm{~m}$.

The same experiments are used to assess the role of the vertical velocity shear and surface motions computing for each cycle $\left|\underline{X}_{\text {Tot }}\right|,\left|\underline{X}_{\text {drift }}\right|$ and the error $\Delta$ on the estimate of the intermediate velocity that is defined as:

$\Delta\left(T_{\text {drift }}\right)=\frac{\| \underline{X}_{\text {Tot }}|-| \underline{X}_{\text {drift }}||}{\left|\underline{X}_{\text {drift }}\right|}$.

This huge statistics is then used to directly quantify the dependence of $\Delta$ on $T_{\text {drift }}$ and to define possible criteria to decrease the error (2) or to identify geographical area where the vertical shear gives raise to smaller error $\Delta$.

In Fig. 4 we show the scatter plot of $\left|\underline{X}_{\text {Tot }}\right|$ vs. $\left|\underline{X}_{\text {drift }}\right|$ computed for each cycle of the six experiments. As is expected, increasing $T_{\text {drif }}$ the plot is less "scattered" and the correlation between $\left|\underline{X}_{\text {drift }}\right|$ and $\left|\underline{X}_{\text {Tot }}\right|$ is more pronounced. However, due to the existence in each experiment of cycles with small $\left|\underline{X}_{\text {drift }}\right|$, the corresponding mean errors are very high, the standard deviation of $\Delta$ largely exceeds its mean value and the probability density function (pdf) $P(\Delta)$ 

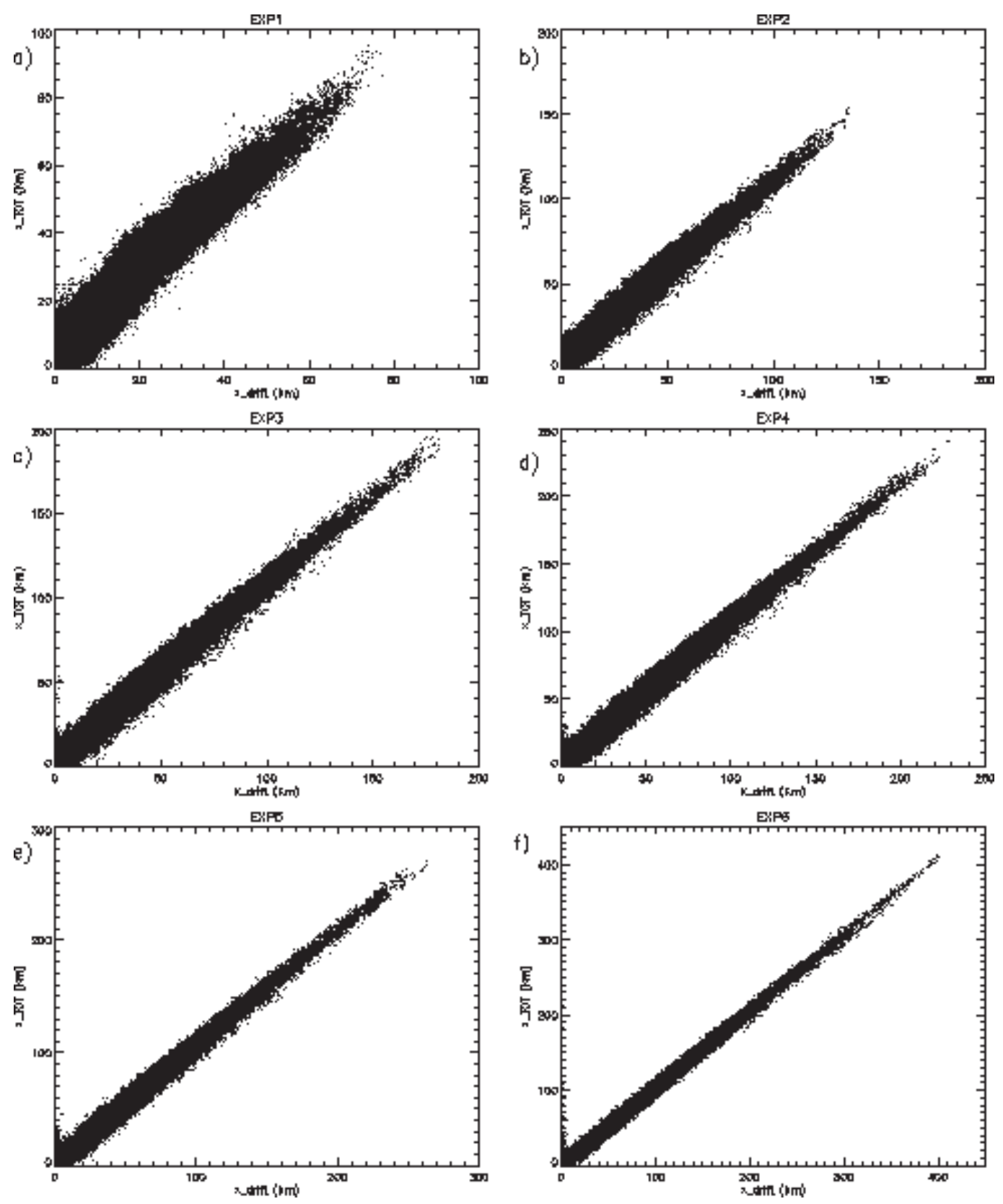

Fig. 4. Scatter plot of $\left|\boldsymbol{X}_{\text {tot }}\right|$ vs. $\left|\boldsymbol{X}_{\text {drift }}\right|$ for EXP1-EXP6 (panels a to f).

of $\Delta$ (Fig. 5), even if characterized by well defined peak ("mode" value) for relatively low values of $\Delta$, has very long tails. Considering only cycles in which the surface displacement $\left|\underline{X}_{\text {Tot }}\right|$ is greater than 10 and $20 \mathrm{~km}$ the estimate on the intermediate velocity definitely improves. In Table 3 we report, for such cycles, mean and standard deviation of $\Delta$ and other statistical indices computed from the cumulative $F(\hat{\Delta})=\int_{0}^{\hat{\Delta}} P(\Delta) d \Delta$, which represents the probability to have a value $\Delta<\hat{\Delta}$. For instance if in EXP2 we consider only cycles with $\left|\underline{X}_{\text {Tot }}\right|>10 \mathrm{~km}(20 \mathrm{~km})$ we have that $50 \%$ of cycles have an error less than $0.25(0.23)$ and that the $37 \%$, $69 \%$ and $86 \%(41 \%, 70 \%$ and $92 \%)$ of cycles gives an estimate of the intermediate velocities with an error smaller than $0.15,0.30$ and $0.50\left(F\left(\Delta_{\text {Mode }}\right), F\left(2 \cdot \Delta_{\text {Mode }}\right)\right.$ and $\left.F(0.5)\right)$, respectively. Neglecting cycles with small $\left|\underline{X}_{\text {Tot }}\right|$ (the only experimental observable variable) is only a first guess in order to attempt to avoid floats with weak intermediate current. In a barotropic situation a small $\left|\underline{X}_{\text {Tot }}\right|$ implies a small $\left|\underline{X}_{\text {drift }}\right|$ and considering only cycles with $\left|\underline{X}_{\text {Tot }}\right|$ greater than a threshold value may help to avoid floats moving very slowly; however, in presence of strong shear it is possible to have small $\left|\underline{X}_{\text {drift }}\right|$ 
Table 3. Different statistical indices from the pdf of the error $(\Delta)$ from the six experiments.

\begin{tabular}{llllllll}
\hline & & $\bar{\Delta} \pm \sigma_{\Delta}$ & $\Delta_{\bmod e}$ & $\Delta_{\text {median }}$ & $F(\Delta \bmod e)$ & $F\left(2 \cdot \Delta_{\bmod e}\right)$ & $F(0.5)$ \\
\hline \multirow{2}{*}{ EXP1 } & $\left|X_{\text {Tot }}\right|>10$ & $0.7 \pm 10$ & 0.350 & 0.52 & 0.33 & 0.73 & 0.47 \\
& $\left|X_{\text {Tot }}\right|>20$ & $0.5 \pm 0.3$ & 0.350 & 0.46 & 0.39 & 0.84 & 0.56 \\
\hline \multirow{2}{*}{ EXP2 } & $\left|X_{\text {Tot }}\right|>10$ & $0.3 \pm 29$ & 0.150 & 0.25 & 0.37 & 0.69 & 0.86 \\
& $\left|X_{\text {Tot }}\right|>20$ & $0.3 \pm 0.2$ & 0.150 & 0.23 & 0.41 & 0.70 & 0.92 \\
\hline EXP3 & $\left|X_{\text {Tot }}\right|>10$ & $0.3 \pm 5$ & 0.100 & 0.17 & 0.44 & 0.73 & 0.94 \\
& $\left|X_{\text {Tot }}\right|>20$ & $0.2 \pm 0.5$ & 0.100 & 0.15 & 0.48 & 0.78 & 0.98 \\
\hline \multirow{2}{*}{ EXP4 } & $\left|X_{\text {Tot }}\right|>10$ & $0.2 \pm 6$ & 0.075 & 0.12 & 0.53 & 0.76 & 0.97 \\
& $\left|X_{\text {Tot }}\right|>20$ & $0.1 \pm 0.8$ & 0.075 & 0.11 & 0.56 & 0.81 & 0.99 \\
\hline \multirow{2}{*}{ EXP5 } & $\left|X_{\text {Tot }}\right|>10$ & $0.3 \pm 14$ & 0.050 & 0.09 & 0.54 & 0.74 & 0.98 \\
& $\left|X_{\text {Tot }}\right|>20$ & $0.1 \pm 5$ & 0.050 & 0.09 & 0.57 & 0.78 & 0.99 \\
\hline \multirow{2}{*}{ EXP6 } & $\left|X_{\text {Tot }}\right|>10$ & $0.4 \pm 20$ & 0.025 & 0.04 & 0.72 & 0.82 & 0.99 \\
& $\left|X_{\text {Tot }}\right|>20$ & $0.3 \pm 17$ & 0.025 & 0.04 & 0.75 & 0.85 & 0.99 \\
\hline
\end{tabular}
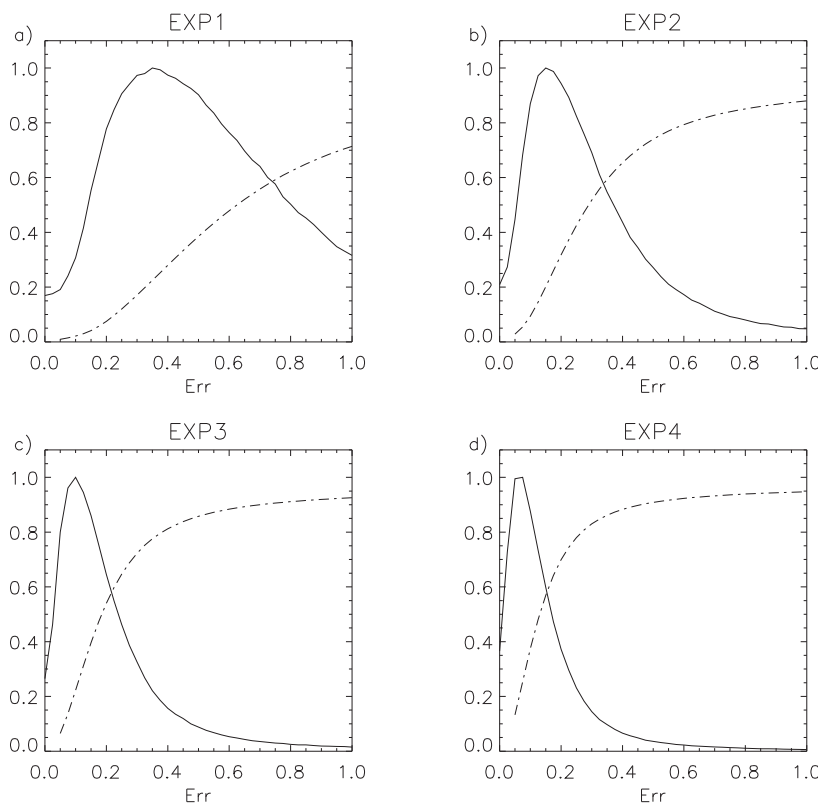

Fig. 5. Pdf of $\Delta$ and its cumulative (dashed-dotted line) computed considering all the cycles for the four experiments EXP1-EXP4.

with large $\left|\underline{X}_{\text {Tot }}\right|$. In Fig. 6 we show the pdf of $\Delta$ together with its cumulative computed considering only cycles with $\left|\underline{X}_{\text {Tot }}\right|>20 \mathrm{~km}$ for the four experiments EXP1-EXP4.

Obviously the error decreases when the time $T_{\text {drift }}$ increases but since the primary aim of the MedARGO experiment was the collection of the maximum number of independent TS vertical profiles, and since the assimilation procedure of positions in an OGGM requires that the parking time $T_{\text {drift }} \leq T_{L}$, in the following we will analyze results only from EXP1 and EXP2. In particular, to identify
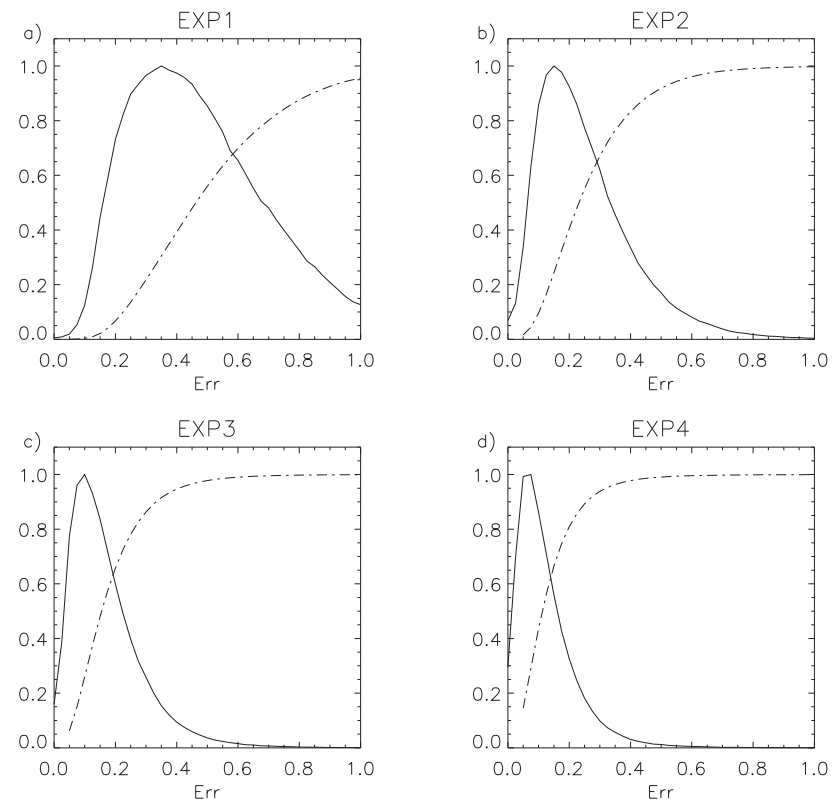

Fig. 6. Pdf of $\Delta$ and its cumulative (dashed-dotted line) computed considering only cycles with $\left|\underline{X}_{\text {Tot }}\right|>20 \mathrm{~km}$ for the four experiments EXP1-EXP4.

a possible dependence of $\Delta$ on the geographical position, we report in Tables 4 and 5 for such experiments the values of $\bar{\Delta} \pm \sigma_{\Delta}, \Delta_{\text {Mode }}, \Delta_{\text {Median }} F\left(\Delta_{\text {Mode }}\right), F\left(2 \cdot \Delta_{\text {Mode }}\right)$ and $F(0.5)$ computed considering cycles with $\left|\underline{X}_{\text {Tot }}\right|>10 \mathrm{~km}$ and $20 \mathrm{~km}$ in the 13 regions indicated in Fig. 2. For cycles with $\left|\underline{X}_{\text {Tot }}\right|>20 \mathrm{~km}$, the standard deviation is smaller than the mean values almost everywhere and for EXP2 $\Delta_{\text {Median }}$ ranges from 0.20 (Regions 3 and 6) to 0.32 (Region 7) and we have that the probability of having an estimate of the 
Table 4. EXP1: $N_{\text {cycles }}$, and statistical indices from the pdf of $\Delta$, in the different regions defined in Fig. 2.

\begin{tabular}{|c|c|c|c|c|c|c|c|c|}
\hline EXP1 & & $N_{\text {cycles }}$ & $\Delta \pm \sigma_{\Delta}$ & $\Delta \bmod e$ & $\Delta_{\text {median }}$ & $F(\Delta \bmod e)$ & $F(2 \cdot \Delta \bmod e)$ & $F(0.5)$ \\
\hline \multirow[t]{2}{*}{ Reg 1} & $\left|X_{\text {Tot }}\right|>10$ & 27498 & $1.4 \pm 8$ & 0.450 & 0.61 & 0.40 & 0.73 & 0.40 \\
\hline & $\left|X_{\text {Tot }}\right|>20$ & 8731 & $0.6 \pm 0.9$ & 0.500 & 0.56 & 0.49 & 0.93 & 0.42 \\
\hline \multirow[t]{2}{*}{$\operatorname{Reg} 2$} & $\left|X_{\text {Tot }}\right|>10$ & 258116 & $0.8 \pm 22$ & 0.375 & 0.54 & 0.35 & 0.74 & 0.44 \\
\hline & $\left|X_{\text {Tot }}\right|>20$ & 97325 & $0.5 \pm 0.3$ & 0.450 & 0.49 & 0.51 & 0.93 & 0.51 \\
\hline \multirow[t]{2}{*}{$\operatorname{Reg} 3$} & $\left|X_{\text {Tot }}\right|>10$ & 272525 & $0.6 \pm 4$ & 0.275 & 0.43 & 0.33 & 0.71 & 0.59 \\
\hline & $\left|X_{\text {Tot }}\right|>20$ & 139494 & $0.4 \pm 0.2$ & 0.225 & 0.38 & 0.29 & 0.70 & 0.70 \\
\hline \multirow[t]{2}{*}{$\operatorname{Reg} 4$} & $\left|X_{\text {Tot }}\right|>10$ & 143850 & $0.8 \pm 11$ & 0.500 & 0.62 & 0.42 & 0.84 & 0.36 \\
\hline & $\left|X_{\text {Tot }}\right|>20$ & 39413 & $0.6 \pm 0.3$ & 0.45 & 0.55 & 0.42 & 0.92 & 0.42 \\
\hline \multirow[t]{2}{*}{ Reg 5} & $\left|X_{\text {Tot }}\right|>10$ & 838 & $0.9 \pm 2$ & 0.375 & 0.66 & 0.26 & 0.62 & 0.33 \\
\hline & $\left|X_{\text {Tot }}\right|>20$ & 101 & $0.6 \pm 0.3$ & 0.475 & 0.55 & 0.48 & 0.89 & 0.46 \\
\hline \multirow[t]{2}{*}{ Reg 6} & $\left|X_{\text {Tot }}\right|>10$ & 145072 & $0.6 \pm 0.7$ & 0.300 & 0.47 & 0.32 & 0.71 & 0.54 \\
\hline & $\left|X_{\text {Tot }}\right|>20$ & 74978 & $0.5 \pm 0.2$ & 0.300 & 0.41 & 0.38 & 0.83 & 0.65 \\
\hline \multirow[t]{2}{*}{ Reg 7} & $\left|X_{\text {Tot }}\right|>10$ & 15209 & $0.9 \pm 1.3$ & 0.575 & 0.72 & 0.40 & 0.81 & 0.28 \\
\hline & $\left|X_{\text {Tot }}\right|>20$ & 2035 & $0.6 \pm 0.4$ & 0.475 & 0.57 & 0.43 & 0.91 & 0.40 \\
\hline \multirow[t]{2}{*}{$\operatorname{Reg} 8$} & $\left|X_{\text {Tot }}\right|>10$ & 337150 & $0.7 \pm 7$ & 0.375 & 0.54 & 0.35 & 0.75 & 0.45 \\
\hline & $\left|X_{\text {Tot }}\right|>20$ & 128512 & $0.5 \pm 0.3$ & 0.425 & 0.48 & 0.48 & 0.92 & 0.52 \\
\hline \multirow[t]{2}{*}{ Reg 9} & $\left|X_{\text {Tot }}\right|>10$ & 30283 & $0.6 \pm 0.6$ & 0.250 & 0.50 & 0.23 & 0.56 & 0.50 \\
\hline & $\left|X_{\text {Tot }}\right|>20$ & 11199 & $0.5 \pm 0.3$ & 0.250 & 0.44 & 0.27 & 0.66 & 0.59 \\
\hline \multirow[t]{2}{*}{ Reg 10} & $\left|X_{\text {Tot }}\right|>10$ & 188400 & $0.6 \pm 3.0$ & 0.375 & 0.51 & 0.37 & 0.80 & 0.48 \\
\hline & $\left|X_{\text {Tot }}\right|>20$ & 93427 & $0.5 \pm 0.2$ & 0.375 & 0.48 & 0.41 & 0.87 & 0.54 \\
\hline \multirow[t]{2}{*}{ Reg 11} & $\left|X_{\text {Tot }}\right|>10$ & 194169 & $0.6 \pm 0.5$ & 0.425 & 0.54 & 0.41 & 0.83 & 0.44 \\
\hline & $\left|X_{\text {Tot }}\right|>20$ & 80365 & $0.5 \pm 0.2$ & 0.425 & 0.51 & 0.45 & 0.91 & 0.49 \\
\hline \multirow[t]{2}{*}{ Reg 12} & $\left|X_{\text {Tot }}\right|>10$ & 188226 & $0.7 \pm 9$ & 0.375 & 0.52 & 0.37 & 0.78 & 0.48 \\
\hline & $\left|X_{\text {Tot }}\right|>20$ & 95655 & $0.5 \pm 0.3$ & 0.325 & 0.46 & 0.34 & 0.80 & 0.56 \\
\hline \multirow[t]{2}{*}{ Reg 13} & $\left|X_{\text {Tot }}\right|>10$ & 107653 & $0.8 \pm 12$ & 0.375 & 0.55 & 0.34 & 0.74 & 0.44 \\
\hline & $\left|X_{\text {Tot }}\right|>20$ & 41827 & $0.5 \pm 0.3$ & 0.425 & 0.49 & 0.47 & 0.92 & 0.52 \\
\hline
\end{tabular}

intermediate velocity with an error $<0.5(\mathrm{~F}(0.5))$ varies from the $80 \%$ (Region 7) to the $95 \%$ (Region 3). This regional analysis shows that the estimate of the intermediate velocities is characterized by a smaller error in the North Western Mediterranean Sea (NWM) in the Northern Ionian (Regions 3 and 6) and in Western Levantine basin (Regions 10 and 11) while the Tyrrhenian and the Adriatic Seas and the Sicily Channel are expected to be characterized by a larger error. In Fig. 7 we map for EXP2 the mean error $\bar{\Delta}$ computed averaging, on a grid of $0.25^{\circ} \times 0.25^{\circ}$, between all the error values of the cycles with $\left|\underline{X}_{\text {Tot }}\right|>10 \mathrm{~km}$ and $\left|\underline{X}_{\text {Tot }}\right|>20 \mathrm{~km}$ falling in a given bin. From this figure we can also see that the whole area north to the African coast (influenced by the flow of the Modified Atlantic Water which gives rise to a strong velocity shear) is characterized by a greater error on the estimate of the intermediate velocities.

Finally, in Fig. 8 we show, always for EXP2, the initial conditions of the numerical ARGO floats characterized by having $\bar{\Delta}<0.25$, where now $\bar{\Delta}$ is the mean error computed on all the cycles of the entire 1 year long trajectory. Except some small scale features, like the spots in vicinity of the Sicily and Sardinia Channel, of difficult explanation, also the large scale picture of this figure indicates that numerical simulations suggest that the NWM, the Ionian Sea together with the Levantine basin are favourite sites for the deployment of ARGO profilers in order to have smaller error on the estimate of sub-surface velocities.

We conclude this section noting that the analysis of numerical profilers other than indicating areas where one can expect a smaller error on the estimate of subsurface velocities were useful to define the parking time $T_{\text {drift }}$ for the real profilers that was finally chosen to be equal to 5 days (Poulain et al., 2006), since this values is as a good compromise between the different and contrasting needs of having the larger number of independent observations, a small error on the estimate of the intermediate velocities and a time length of the profiler cycle smaller than the intermediate velocities decorrelation time $T_{L}$. 
Table 5. EXP2: $N_{\text {cycles }}$, and different statistical indices from the pdf of $\Delta$, in the different regions defined in Fig. 2.

\begin{tabular}{|c|c|c|c|c|c|c|c|c|}
\hline EXP2 & & $N_{\text {cycles }}$ & $\Delta \pm \sigma_{\Delta}$ & $\Delta \bmod e$ & $\Delta_{\text {median }}$ & $F(\Delta \bmod e)$ & $F(2 \cdot \Delta \bmod e)$ & $F(0.5)$ \\
\hline \multirow[t]{2}{*}{ Reg 1} & $\left|X_{\text {Tot }}\right|>10$ & 26067 & $0.8 \pm 7.8$ & 0.125 & 0.30 & 0.29 & 0.50 & 0.73 \\
\hline & $\left|X_{\text {Tot }}\right|>20$ & 14517 & $0.3 \pm 0.3$ & 0.175 & 0.26 & 0.43 & 0.74 & 0.83 \\
\hline \multirow[t]{2}{*}{ Reg 2} & $\left|X_{\text {Tot }}\right|>10$ & 185585 & $0.4 \pm 7.2$ & 0.175 & 0.25 & 0.43 & 0.75 & 0.84 \\
\hline & $\left|X_{\text {Tot }}\right|>20$ & 116408 & $0.3 \pm 0.2$ & 0.150 & 0.24 & 0.40 & 0.75 & 0.91 \\
\hline \multirow[t]{2}{*}{ Reg 3} & $\left|X_{\text {Tot }}\right|>10$ & 185440 & $0.3 \pm 3.4$ & 0.125 & 0.21 & 0.39 & 0.70 & 0.91 \\
\hline & $\left|X_{\text {Tot }}\right|>20$ & 132768 & $0.2 \pm 0.2$ & 0.125 & 0.20 & 0.43 & 0.76 & 0.95 \\
\hline \multirow[t]{2}{*}{$\operatorname{Reg} 4$} & $\left|X_{\text {Tot }}\right|>10$ & 107209 & $0.4 \pm 2.4$ & 0.175 & 0.31 & 0.32 & 0.67 & 0.80 \\
\hline & $\left|X_{\text {Tot }}\right|>20$ & 53569 & $0.3 \pm 0.2$ & 0.175 & 0.28 & 0.36 & 0.76 & 0.88 \\
\hline \multirow[t]{2}{*}{$\operatorname{Reg} 5$} & $\left|X_{\text {Tot }}\right|>10$ & 532 & $0.5 \pm 1.8$ & 0.150 & 0.30 & 0.32 & 0.59 & 0.79 \\
\hline & $\left|X_{\text {Tot }}\right|>20$ & 181 & $0.3 \pm 0.2$ & 0.150 & 0.25 & 0.41 & 0.66 & 0.90 \\
\hline \multirow[t]{2}{*}{ Reg 6} & $\left|X_{\text {Tot }}\right|>10$ & 98561 & $0.3 \pm 1.4$ & 0.150 & 0.22 & 0.44 & 0.76 & 0.89 \\
\hline & $\left|X_{\text {Tot }}\right|>20$ & 69176 & $0.2 \pm 0.1$ & 0.150 & 0.20 & 0.49 & 0.83 & 0.94 \\
\hline \multirow[t]{2}{*}{ Reg 7} & $\left|X_{\text {Tot }}\right|>10$ & 11082 & $0.5 \pm 5.1$ & 0.175 & 0.35 & 0.27 & 0.59 & 0.71 \\
\hline & $\left|X_{\text {Tot }}\right|>20$ & 4116 & $0.4 \pm 0.3$ & 0.275 & 0.32 & 0.51 & 0.89 & 0.80 \\
\hline \multirow[t]{2}{*}{ Reg 8} & $\left|X_{\text {Tot }}\right|>10$ & 241273 & $0.3 \pm 1.1$ & 0.175 & 0.26 & 0.41 & 0.75 & 0.85 \\
\hline & $\left|X_{\text {Tot }}\right|>20$ & 151746 & $0.3 \pm 0.2$ & 0.175 & 0.24 & 0.45 & 0.82 & 0.91 \\
\hline \multirow[t]{2}{*}{ Reg 9} & $\left|X_{\text {Tot }}\right|>10$ & 19822 & $0.3 \pm 0.4$ & 0.150 & 0.25 & 0.38 & 0.68 & 0.84 \\
\hline & $\left|X_{\text {Tot }}\right|>20$ & 13144 & $0.3 \pm 0.2$ & 0.150 & 0.22 & 0.44 & 0.76 & 0.92 \\
\hline \multirow[t]{2}{*}{ Reg 10} & $\left|X_{\text {Tot }}\right|>10$ & 121976 & $0.3 \pm 3.5$ & 0.150 & 0.25 & 0.36 & 0.73 & 0.89 \\
\hline & $\left|X_{\text {Tot }}\right|>20$ & 93004 & $0.3 \pm 0.1$ & 0.175 & 0.23 & 0.47 & 0.84 & 0.93 \\
\hline \multirow[t]{2}{*}{ Reg 11} & $\left|X_{\text {Tot }}\right|>10$ & 129813 & $0.3 \pm 0.2$ & 0.175 & 0.26 & 0.39 & 0.76 & 0.87 \\
\hline & $\left|X_{\text {Tot }}\right|>20$ & 93328 & $0.3 \pm 0.1$ & 0.175 & 0.25 & 0.43 & 0.82 & 0.91 \\
\hline \multirow[t]{2}{*}{ Reg 12} & $\left|X_{\text {Tot }}\right|>10$ & 122763 & $0.3 \pm 0.7$ & 0.150 & 0.25 & 0.36 & 0.71 & 0.87 \\
\hline & $\left|X_{\text {Tot }}\right|>20$ & 91741 & $0.3 \pm 0.2$ & 0.150 & 0.23 & 0.40 & 0.77 & 0.92 \\
\hline \multirow[t]{2}{*}{ Reg 13} & $\left|X_{\text {Tot }}\right|>10$ & 70432 & $0.4 \pm 9.3$ & 0.175 & 0.26 & 0.42 & 0.74 & 0.83 \\
\hline & $\left|X_{\text {Tot }}\right|>20$ & 46665 & $0.3 \pm 0.2$ & 0.175 & 0.23 & 0.47 & 0.82 & 0.91 \\
\hline
\end{tabular}

\section{Numerical surface particles}

We present here the results related to the study of the interannual variability of the surface Lagrangian transport in two key areas of the Mediterranean Sea obtained utilizing the MFSPP archive of Eulerian velocity fields from 2000 to 2004. Lagrangian trajectories were computed using the off-line ARIANE algorithm (Blanke and Raynaud, 1997, http://www.univ-brest.fr/lpo/ariane) in which, to mimic the behaviour of floating objects, numerical particles are kept at the sea surface imposing null vertical velocity.

3.1 Interannual variability of the Lagrangian transport in the Corsica and Sardinia Channels

In the Sardinia Channel the near surface Modified Atlantic Water (MAW) flows eastward and, in proximity of Sicily, bifurcates in two branches, one entering the Tyrrhenian Sea, the other flowing in the Eastern Mediterranean (EM) through the Sicily Channel (e.g., Astraldi et al., 1999). The MAW entering the Tyrrhenian Sea participates to its cyclonic surface circulation and it is subject to a further bifurcation (Artale et al., 2006) in two branches, one recirculating in the Sardinia Channel, the other entering the Ligurian Sea through the Corsica Channel (approximately $400 \mathrm{~m}$ deep) that is characterized by an highly variable, barotropic, northward flow of both surface water of Atlantic origin and intermediate water of eastern origin (Astraldi and Gasparini, 1992). The study of the interannual variability of the path of the relatively fresher MAW in these two key areas is an important issue since both in the North Western Mediterranean (NWM) and in the EM occur deep water formation processes (among others MEDOC, 1970; Robinson and Golnaraghi, 1994; Mertens and Schott, 1998; Malanotte-Rizzoli et al., 1999), that are highly influenced by the surface water salinity.

Using the one day averaged MFSPP Eulerian velocity fields from 2000 to 2004 we perform two experiments (CORS and SARD) in which starting from January 2000 we release every week, respectively, 12354 and 11980 particles in the two 1-grid thick sections covering the Corsica and Sardinia Channels (see Fig. 9). Such particles are then 

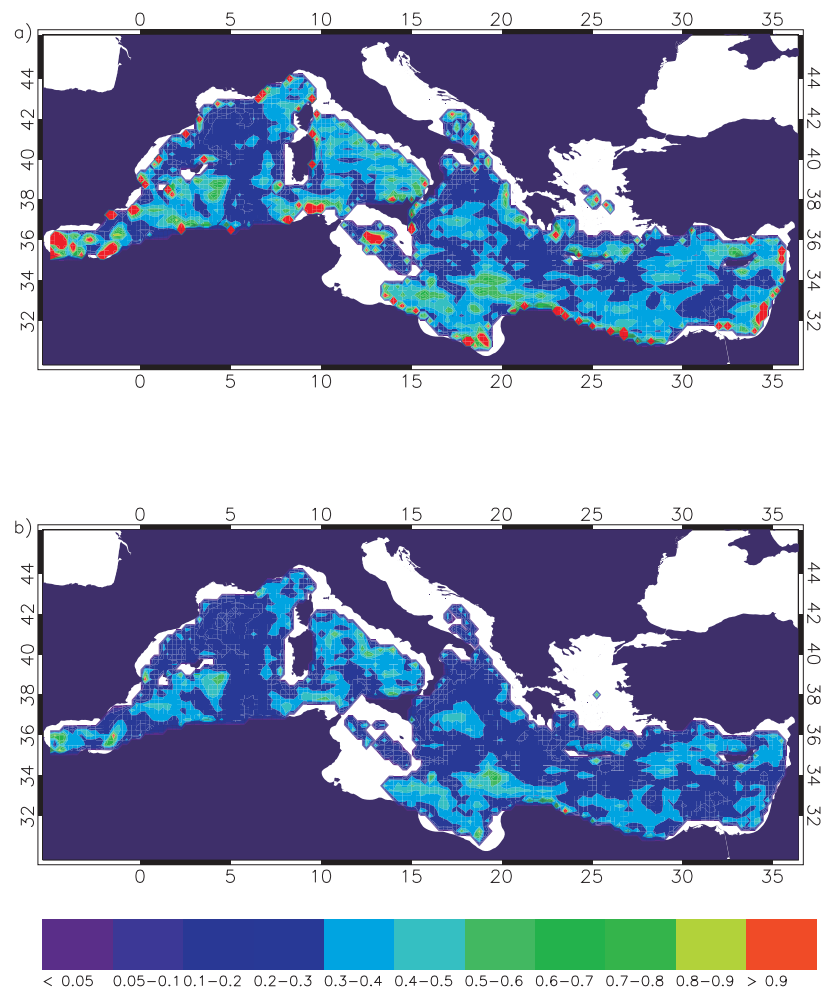

Fig. 7. EXP2: maps of the mean error $\bar{\Delta}$ computed averaging on a grid of $0.25^{\circ} \times 0.25^{\circ}$ between all the error values of the cycles falling in a given bin with $\left|\underline{X}_{\text {Tot }}\right|>10 \mathrm{~km}$ (panel a) and $\left|\underline{X}_{\text {Tot }}\right|>20 \mathrm{~km}$ (panel b).

integrated for 28 days for a total of 258 Lagrangian realizations. In experiment CORS particles are stopped if they recirculate in CORS or if they reach the section LIG while in experiment SARD they are stopped when arriving at the sections TYR and SIC or if they recirculate in SARD. We then construct a time series representing the variability of the surface section to section Lagrangian transport directly computing for each Lagrangian integration (realization) the number of particles that, after a given time, reach the ending sections. Some practical application may require the knowledge of the total integrated quantity of the tracer that reaches a given area in a given time. Consequently, in Fig. 10 we show as a function of time the percentage of particles that reach the section LIG starting from section CORS after 28, 14 and 7 days (panels $a, b$ and c). The thick black line represents the yearly averaged percentage, while the thick red line is the percentage of realizations in which none of the particles reaches the ending section. The Lagrangian flow from the Corsica Channel toward the Western Ligurian Sea shows a well defined and realistic seasonality (Astraldi and Gasparini, 1992) with maximum values in winter when from 2000 to 2003 for extended periods more than $80 \%$ of particles enters the Ligurian Sea in 28 days while few of them are able to arrive, during isolated events, in less than one week at the ending section LIG (Fig. 10c). From 2000 to 2004 the mean flow shows a decreasing trend and the yearly averaged percentage of particles reaching the LIG Section in 28 days (panel a) monotonically decreases from the $52 \%$ in 2000 to about $30 \%$ in 2004 . This tendency is not evident for the faster isolated events while for particles that arrive at the ending section in 14 days the percentage varies from about $20 \%$ for 2000 and 2001 to about $15 \%$ for 2004 . It is interesting to observe that during these 5 years the number of realizations in which none of the particles released in the Corsica Channel reaches the Ligurian Sea (red curves) in 14 and 28 days increases almost monotonically and that during 2003 and 2004 for a period of 4-5 months (summer and beginning of autumn) do not exist a surface flow connecting the Tyrrhenian and the Ligurian basin in less than 28 days. These results agree with experimental observations from surface drifters released in the Tyrrhenian Sea in the context of the Italian project "Ambiente Mediterraneo" (http://clima.casaccia.enea.it/murst/index.html). In particular, during 200326 drifters were deployed in the central part of the basin and only 2 of them (released in the late autumn) were able to exit the Tyrrhenian, entering the Ligurian Sea (Rinaldi, 2006).

In Figs. 11 and 12 we show, respectively, the analogous plots for the surface flow connecting the Sardinia Channel to the Tyrrhenian Sea (Section TYR in Fig. 9) and the EM (Section SIC in Fig. 9). In both cases it cannot be observed a clear seasonality of the signal, even if the isolated events of fast particles reaching the EM in less than one week are mainly concentrated in the winter months (panel $\mathrm{c}$ of Fig. 12). In accord to the CORS experiment, even the surface flow entering the Tyrrhenian Sea from the Sardinia Channel shows a general decreasing tendency from 2000 to 2004 and the percentage of particles that reach the TYR Section in 28 days varies from about $30 \%$ in 2000 to about $15 \%$ in 2004 . The same behaviour is not observed in the surface flow entering the EM (Fig. 12) that shows a maximum value during 2002 in which more than $45 \%$ of particles reaches the ending section SIC. In this year the number of particles entering the EM in 28 days is almost five times the number of particles entering the Tyrrhenian Sea, as it is possible to see in panel (a) of Fig. 13 where is plotted the ratio between the yearly averaged percentage of particles entering the Tyrrhenian and the EM. For slow particles (red and black lines in Fig. 13a) the mean value of this ratio shows a rather high variability around its mean value (about $0.6-0.7$ ) that is very similar to the estimate obtained both from experimental data and numerical simulation (e.g., Herbaut et al., 1996; Pierini et al., 2001). On the contrary, the number of fast particles is definitively larger for the flow connecting the Sardinia Channel to the EM (line blue in panel a of Fig. 13), since only in few (5) Lagrangian realizations particles released in the Sardinia Channel are able to reach the TYR section in less than one week (panel c of Fig. 11). Finally, it is interesting to note that even if the ratio between the yearly averaged percentage 


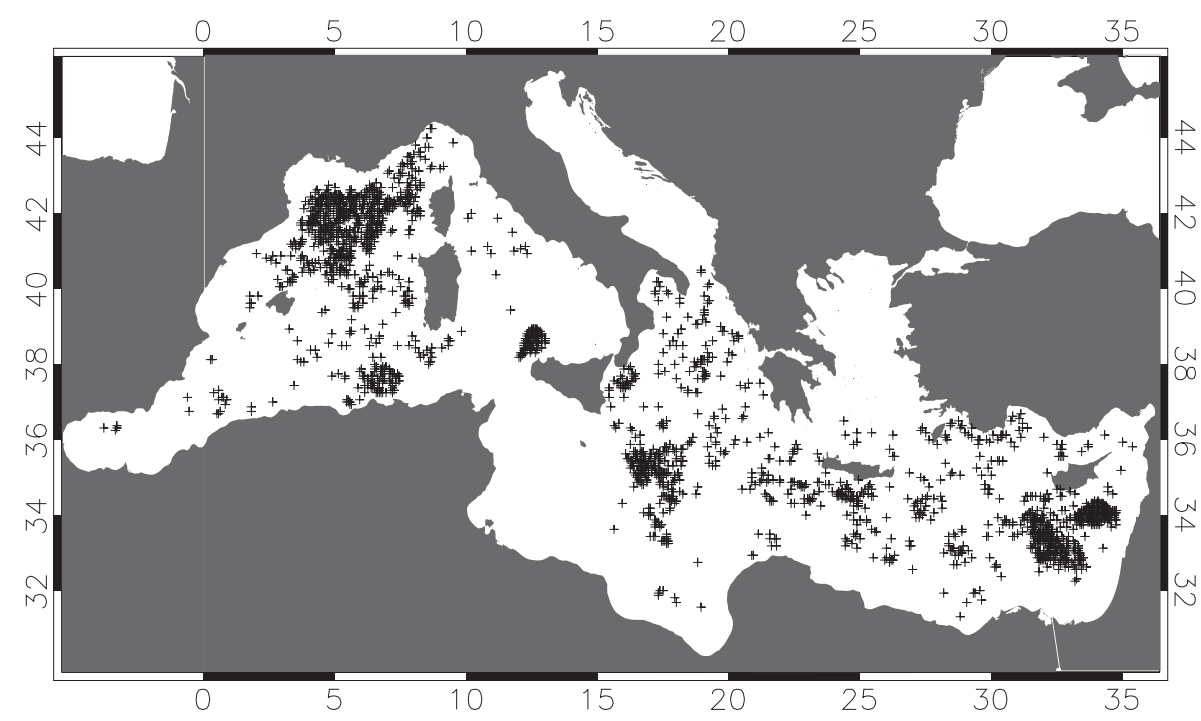

Fig. 8. EXP2: initial conditions of numerical profilers characterized by having the mean error $\bar{\Delta}<0.25$.

of particles arriving at TYR and SIC in less than 28 and 14 days is almost always smaller than one (except than in 2000), we may observe the presence of a non negligible number of Lagrangian realizations in which most of the particles enters the Tyrrhenian Sea as is evident from panels (b) and (c) of Fig. 13 where for each realization is plotted the ratio between the particles reaching the TYR and the SIC section.

Other interesting information may be obtained from the arrival times in the ending sections. Considering all the Lagrangian integrations, we may construct a five years long time series representative of the time behaviour of the parameter in which we are interested. In fact, depending upon the practical application, one may be interested in different parameters of the probability density function (pdf) $P(t)$ of the arrival times in the ending section. For instance, if we are interested in the dispersion of a dangerous pollutant we would like to know when it reaches for the first time a given location. Contrastingly, if we are interested to know for how much time a source of pollution contaminates a given location, we are interested in studying the extreme tail of the arrival times pdf, or, for the practical cases in which the concentration of the tracer is an important factor, the relevant parameter to be considered is the mode value of the pdf.

As an example of a possible application, we plot in Fig. 14 the yearly averaged minimum times (time in which the first particle reaches the ending section, panel a), the mean and the median values of the arrival times to the ending section (panels $\mathrm{c}$ and d). It has to be stressed that, since these characteristic times are computed considering only particles reaching the final sections, the interpretation of their time behavior has to be weighted with the results shown in Figs. 1012 , where we explicitly plot the percentage of realizations in which none of the particles reach the ending sections. From Fig. 14 it is possible to observe that the characteristics times

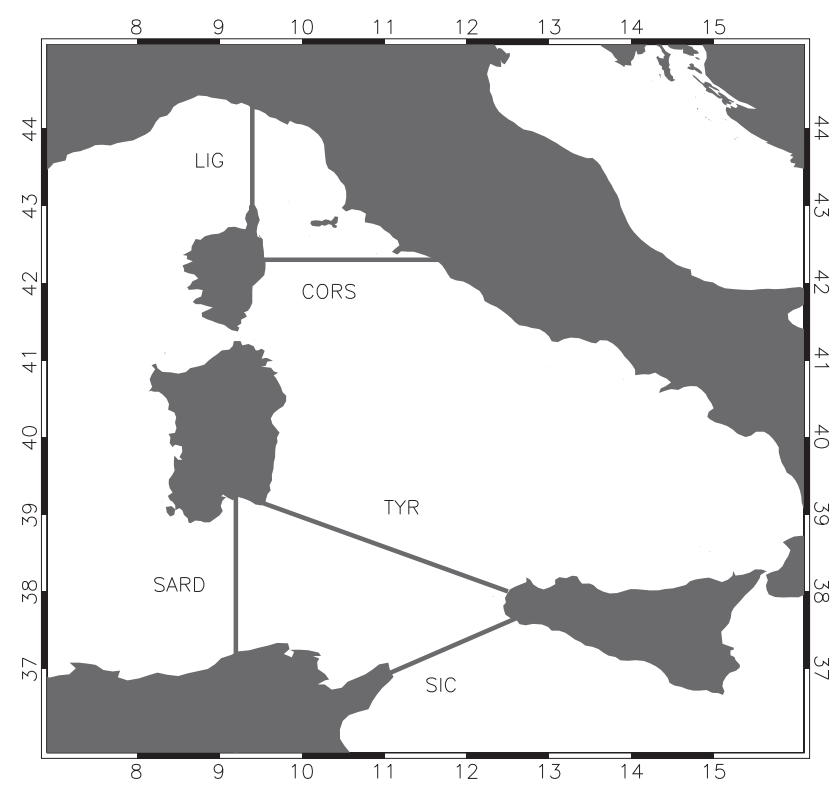

Fig. 9. Starting (SARD and CORS) and ending sections for the Lagrangian experiments described in Sect. 3.

of the transport to the SIC and LIG (red and blue lines) sections oscillates around their mean values (please note that in the CORS experiment the number of particles that do not reach the ending section strongly increases in 2003 and 2004) while all the characteristics times of the surface Lagrangian transport from the Sardinia Channel to the Tyrrhenian Sea (black line) show a tendency to increase during these years of about a $30 \%$. 

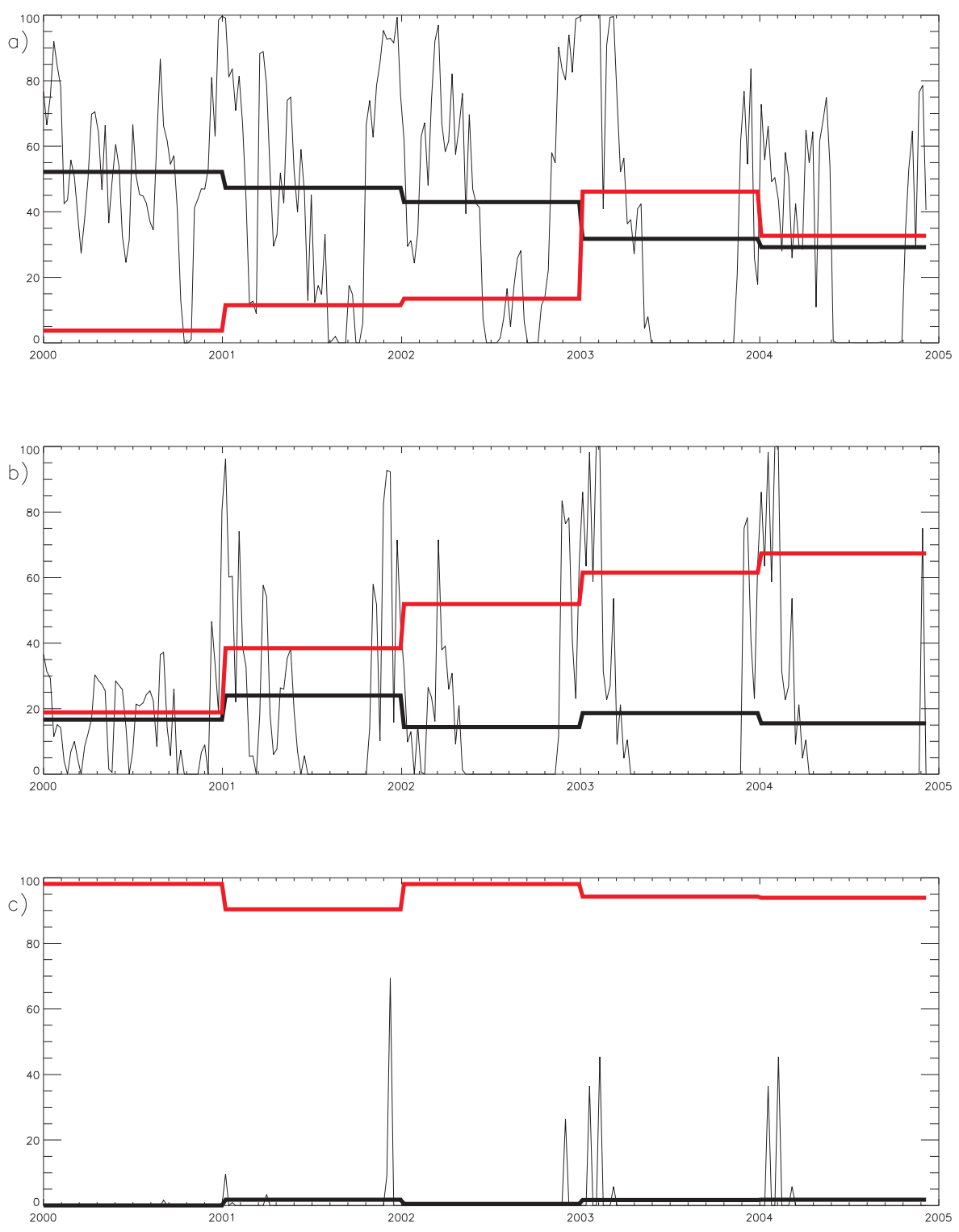

Fig. 10. Percentage of particles reaching, for each Lagrangian realization, the ending section LIG in the CORS Experiment. The thick black line represents the yearly averaged mean. The red thick line represents the yearly averaged percentage of realization in which no particles reach the ending section. In panels (a), (b) and (c) are reported percentage values computed at 28, 14 and 7 days.

\subsubsection{Exponentially decaying particles}

The variability of the characteristic times of the Lagrangian transport may have important consequences when considering tracers which concentration decays with time. A chemical tracer may be subjected to evaporation and also to changes in its buoyancy characteristics. For instance, oil evaporation rate is a rather complex function of time strongly depending from its quality and type. In another context, taking into account a "mortality" rate of particles is crucial in the assessment of the larval exchange among marine communities, an important scientific issue, also for the manage- ment of the fishery stocks and marine reserves. In fact, most marine species have a larval stage and, in particular, planktotrophic larvae are subjected to a wide dispersal since they may drift in the photic zone for a time varying from ten days to two months (Siegel et al., 2003). During this period larvae are subjected to a rate of mortality, depending on variable resources and predation processes, that is usually represented by a constant coefficient, ranging from $\mathrm{O}(1)$ to $\mathrm{O}(30$ days) (Cowen et al., 2000), which leads to an exponentially decay of their concentration.

When dealing with practical applications, it is then essential to take into account the time dependency of the 

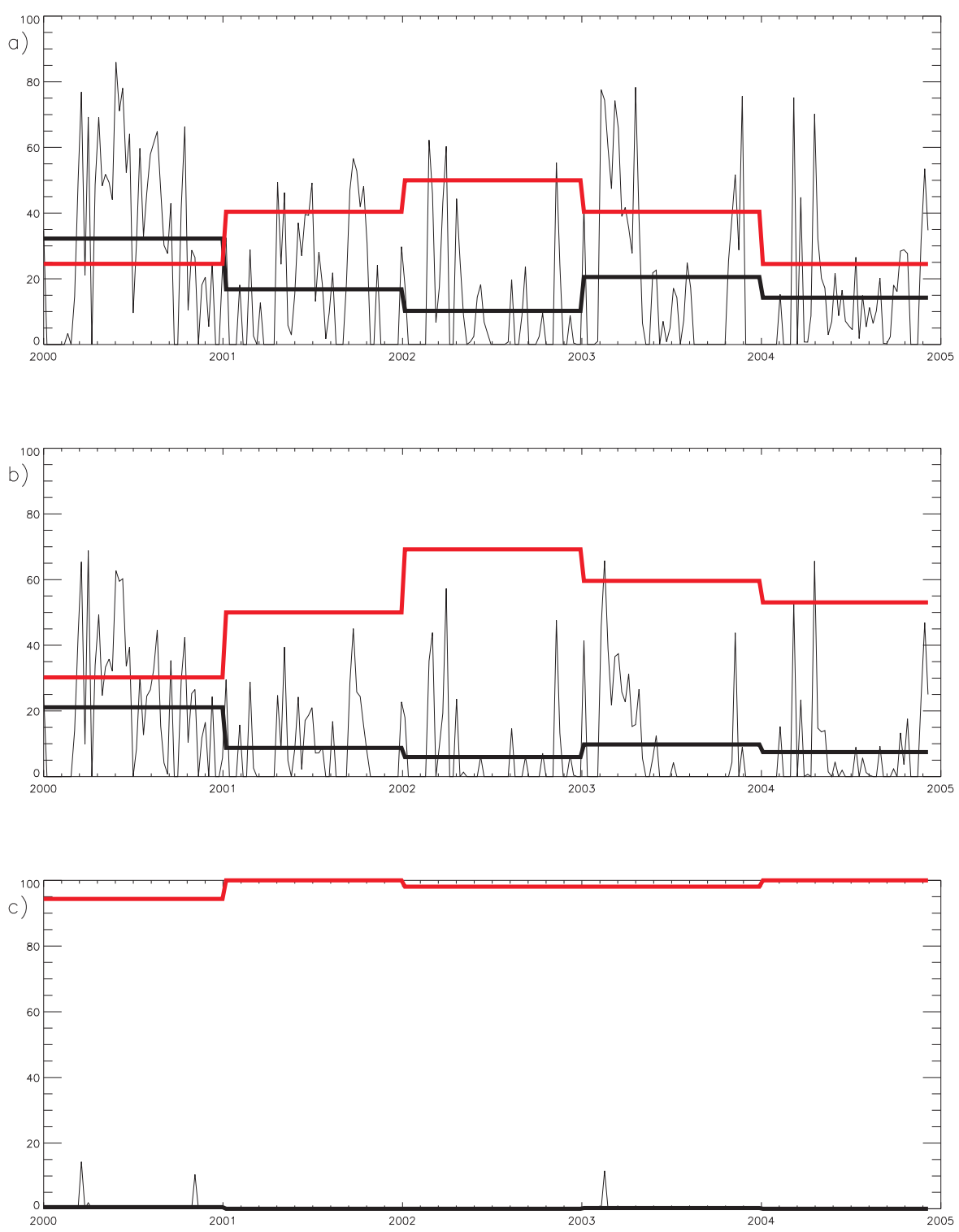

Fig. 11. Percentage of particles reaching, for each Lagrangian realization, the ending section TYR in the SARD Experiment. The thick black line represents the yearly averaged mean. The red thick line represents the yearly averaged percentage of realization in which no particles reach the ending section. In panels (a), (b) and (c) are reported percentage values computed at 28, 14 and 7 days.

concentration of the biological or chemical element we consider. Without entering in the details of the different tracer's phenomenology, we consider here the simplest case in which the advected particles are subjected to a "mortality" represented by the exponential decay $e^{\frac{-t}{\tau}}$ with constant folding time $\tau$. Assuming that the "mortality" affects particles homogeneously and independently of their location we may directly compute, for each Lagrangian integration, the pdf $\hat{P}_{\tau}(t)$ of the arrival times of particles representative of a tracer with concentration decaying with the e-fold time $\tau$ by:

$$
\hat{P}_{\tau}(t)=P(t) \cdot e^{\frac{-t}{\tau}}
$$

where $P(t)$ is the single Lagrangian realization pdf of the arrival time for particles no subjected to mortality. For each realization, the number of the particles reaching the ending section is given by:

$$
\hat{N}_{\tau}(t)=\int_{0}^{t} \hat{P}_{\tau}\left(t^{\prime}\right) d t^{\prime}=\int_{0}^{t} P\left(t^{\prime}\right) \cdot e^{\frac{-t^{\prime}}{\tau}} d t^{\prime} .
$$

In the limiting case of $N(\tilde{t}) \rightarrow \delta(\tilde{t}), \hat{N}_{\tau}(\tilde{t})$ is equal to $N \cdot e^{\frac{-\tilde{t}}{\tau}}$, where $N$ is the number of particles. As an example of application, we compute, for each Lagrangian realization of the two previously described experiments, $\hat{N}_{\tau}(t)$ with 

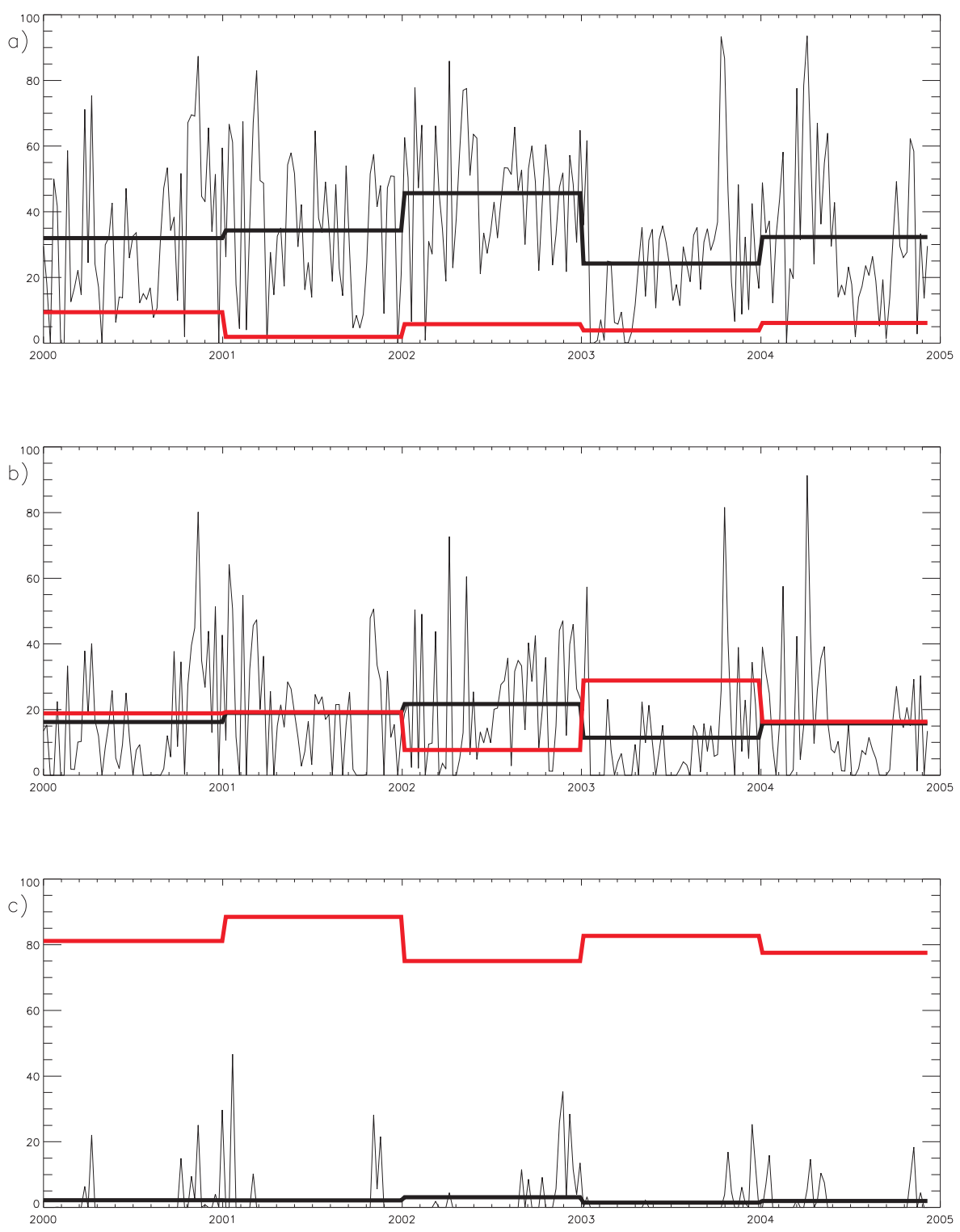

Fig. 12. Percentage of particles reaching, for each Lagrangian realization, the ending section SIC in the SARD Experiment. The thick black line represents the yearly averaged mean. The red thick line represents the yearly averaged percentage of realization in which no particles reach the ending section. In panels (a), (b) and (c) are reported percentage values computed at 28, 14 and 7 days.

$\tau$ equal to 7 and 14 days. Technically, for each time step, the number of particles reaching the ending section is multiplied by the decaying factor, i.e., for $T=M \Delta t$ : $\hat{N}_{\tau}(M \Delta t)=\sum_{i=1}^{M} N(i \Delta t) e^{\frac{-i \Delta t}{\tau}}$.

In Fig. 15 we show the yearly averaged percentages of particles reaching the ending sections for $\tau=7,14$ days (black and red curves) and for the integration time $t=7,14$ and 28 days (dashed, thin and thick curves). These plots, when considered in specific cases, may have an interest per se; here we restrict our analysis to some general comments noting that the percentage of particles, characterized by a given mortality rate arriving to the ending section is linked to the char- acteristic times of the surface "section to section" transport shown in Fig. 14. For instance we may note that, contrastingly with the monotonically decreasing 28-days transport in section LIG shown in panel (a) of Fig. 10 (black curve), when we consider particles with decaying e-fold rate $\tau=7$ days we have, e.g., (Fig. 15a) that the transports in 2003 are greater than in 2002, since in this year (2002) the characteristics times of the transport are greater, as is possible to see from Fig. 14 (blue line). The same is true for the transport in TYR where the (non monotonic decrease) of transport (Fig. 11) is amplified (of about the $50 \%$ for, e.g. for $\tau=14$ and $t=14$, Fig. 15b), when considering particles 

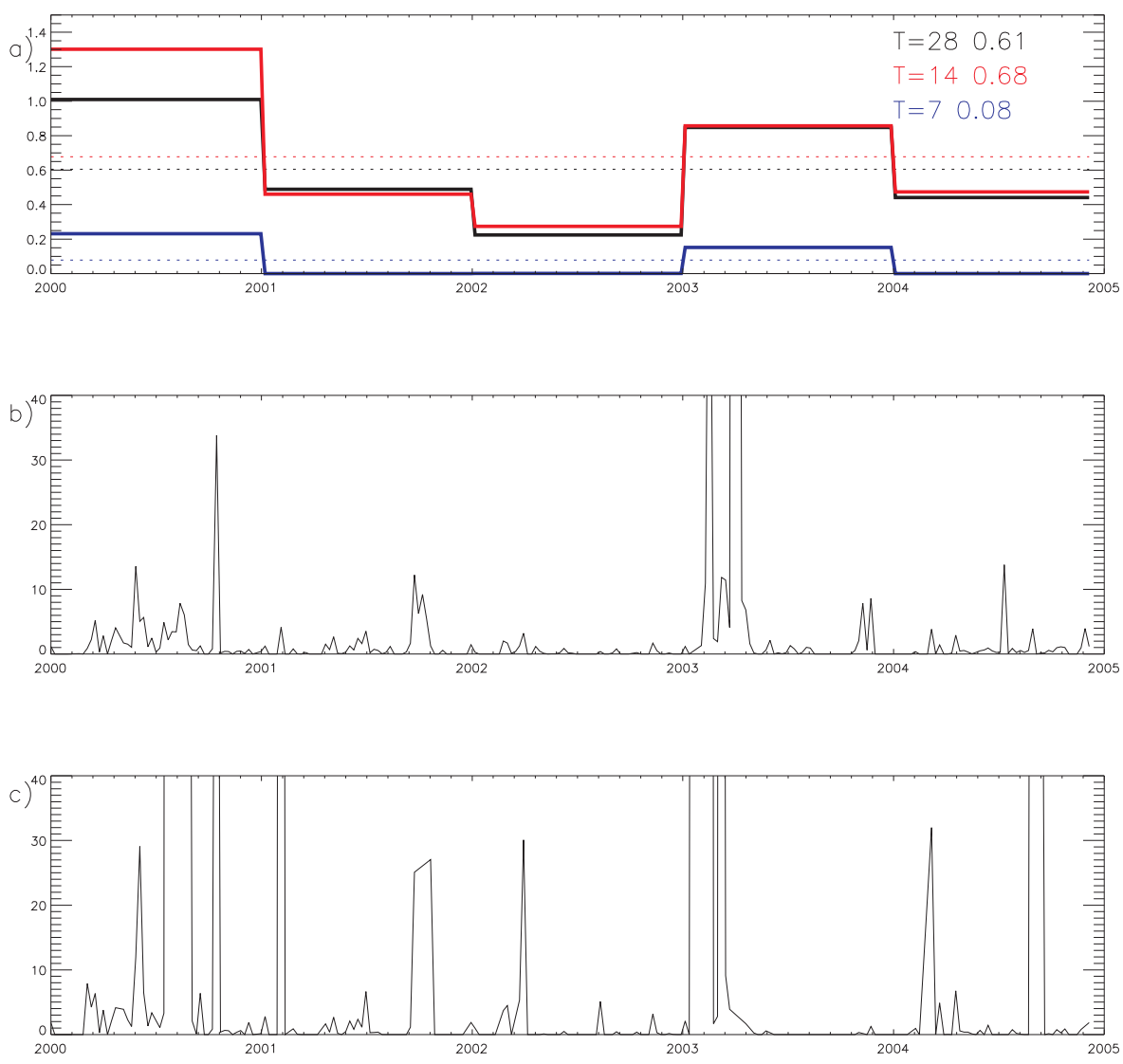

Fig. 13. Panel (a): Ratio between the yearly averaged percentage of particles reaching the TYR and SIC Section in the SARD Experiment after 28, 14 and 7 days (black, red and blue lines, respectively). The dashed line represent the 5 years average, which values is reported in the upper left number. In panels (b) and (c) are reported the time series of the same ratio computed for each realization in which at least one particle reach the SIC section (250 and 209 realizations for integration of 28 and 14 days, respectively in panel b and c).

characterized by a given mortality rate, due to the fact that the characteristic times of the transport from the SARD and TYR section monotonically increase from 2000 to 2004 (Fig. 14). These simple qualitative relations, obviously depending on the space and time scales, may have important and quantitative consequences in practical applications as, e.g., in the dynamics of the marine biota where often the migration and the survival of specific specie critically depend on the concentration of its larvae.

\section{Summary and perspectives}

In this work we have presented some results obtained during MFSTEP by means of numerical simulations of ARGO profilers (Sect. 2) and surface floating objects (Sect. 3).

Simulations of ARGO floats were used to define the optimal time cycling characteristics of the profilers to maximize independent observations of TS vertical profiles and to study the dependence of the error on the estimate of the intermediate velocity as a function of the parking time $T_{\text {drift }}$ and of the geographical areas. The obtained results have suggested the choice $T_{\text {drift }}=5$ days as a good compromise between the different and contrasting needs of having the larger number of independent observations, a small error on the estimate of the intermediate velocities and a time length of the profiler cycle smaller than the intermediate velocities decorrelation time $T_{L}$. Moreover, numerical simulations suggest that in the NWM, in the Ionian Sea and in the Levantine basin one can expect a smaller error on the estimate of subsurface velocities.

The MFSPP archive of the hindcast Eulerian velocity fields was used to construct a surface Lagrangian archive systematically integrating numerical particles released and constrained to drift at surface. In Sect. 3 we presented some results, concerning the study of the interannual variability of the surface dispersion that was obtained from this huge Lagrangian atlas. One of the main assumption of this approach is that the hindcast numerical velocity fields from MFS forecasting model, which assimilate in-situ real data and are forced by high-resolution reanalyzed wind fields, represent the best basin-scale description available for eddy variability and quasi-steady circulation patterns. This trajectories 

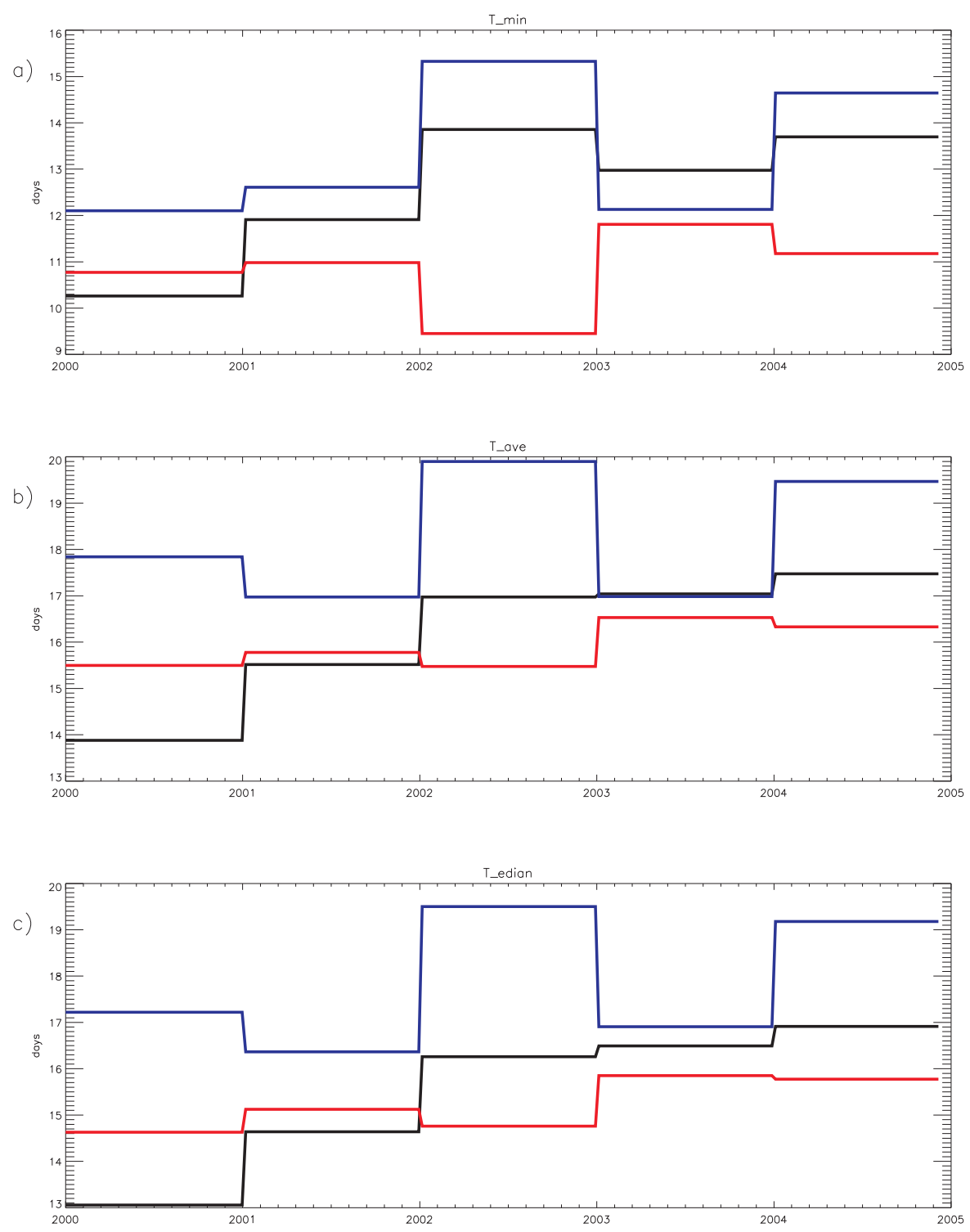

Fig. 14. From panels (a) to (c): yearly averaged values of the minimum values in which the particles reach the ending section ( $T_{-}$min),

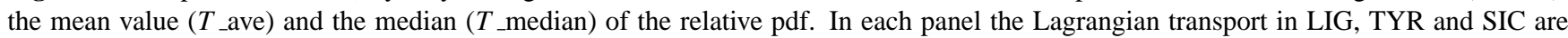
represented by blue, black and red lines.

data set was already used to build, following a statistical approach, seasonal maps of the surface dispersion (Pizzigalli et al., 2007). Here we have provided a further example of exploitation of this Lagrangian data set investigating the interannual variability of the surface Lagrangian transport in two key areas of the Western Mediterranean, the Sardinia and the Corsica Channels. The "section-to section" Lagrangian transport was computed in a relatively short range of time (28 days) since this is the time scale of interest of most of possible practical applications. Lagrangian numerical simulations suggest a monotonic decreasing trend from 2000 to 2004 of the surface flow connecting the Tyrrhenian Sea to the NWM. Moreover they show that during summer and beginning of autumn of 2003 and 2004, for an extended periods of 4-5 months, no particles are able to enter the Ligurian Sea in less than 28 days. These results suggest that the variability of the surface MAW flow from the Tyrrhenian has to be considered as a one of the possible cause of the variability of the hydrological properties observed in the NWM (Herbaut et al., 1997). On the other hand, the surface flow from the Sardinia Channel shows a rather high variability of the ratio between the yearly averaged percentage of particles that reach the Tyrrhenian Sea and the EM around its mean value that, for relatively slow particles results to be about 

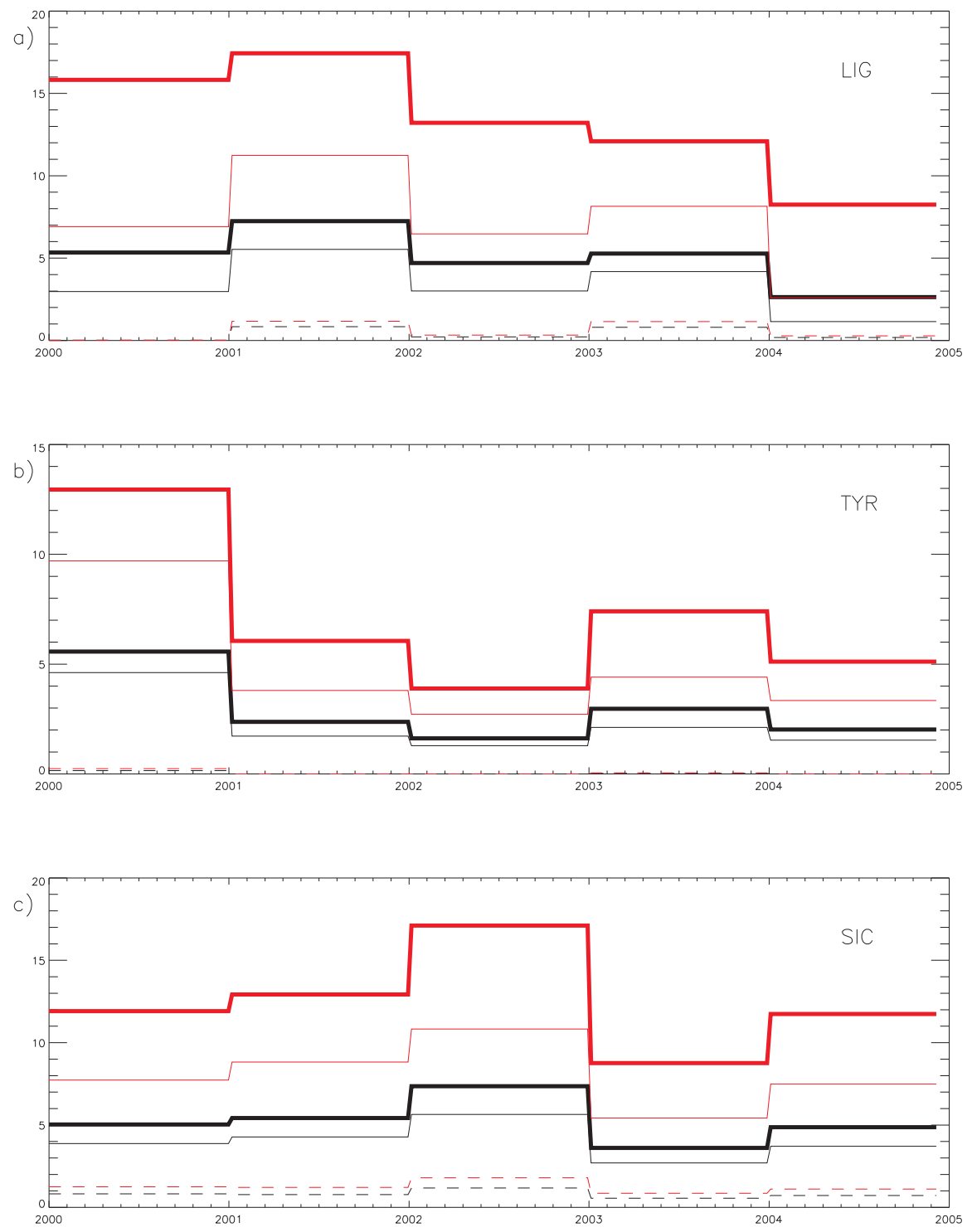

Fig. 15. Yearly averaged percentages of particles for the three ending sections for $\tau=7,14$ days (black and red curves) and for the integration time $t=7,14$ and 28 days (dashed, thin and thick curves). Transports in LIG, TYR and SIC are shown in panels (a), (b) and (c), respectively.

0.6-0.7. However, the Lagrangian analysis shows the presence of a non negligible number of Lagrangian realizations in which most of the particles enter the Tyrrhenian Sea, probably due to the wind forcing. An interesting development of the analysis presented here that definitively could improve the knowledge of the phenomenology of the surface dispersion relies in a systematic analysis of the correlation between wind regimes and path of advected numerical particles.

Finally we have computed the Lagrangian surface transport between the same sections considering particles characterized by an inner rate of mortality. The results shown have to be considered solely as an example of a little step toward more specific applications regarding chemical tracers or biological material dispersal. Further steps toward more realistic applications could be obtained refining this approach considering, e.g., the dispersal of classes of planktotrophic larvae of specific interest for the Mediterranean Sea with a rate mortality depending on the particle paths and on the hydrological properties, or inserting a prescribed downward vertical velocity representative of the change of the buoyancy of the tracer.

In general, we believe that the results shown are encouraging since they show a methodology useful to attain a better knowledge of the surface dispersion properties in the Mediterranean Sea through a simple integration of the MFS Eulerian velocity fields. In particular it could be of possible interest to insert, after having selected some key areas of the Mediterranean where compute the Lagrangian 
transport on such time scale $(\mathrm{O}($ month $))$, this kind of analysis in the Mediterranean monthly bulletin (http://www.bo.ingv. $\mathrm{it} / \mathrm{mfs} / \mathrm{monthly.htm)} \mathrm{provided} \mathrm{by} \mathrm{now} \mathrm{by} \mathrm{the} \mathrm{Italian} \mathrm{Group}$ of Operational Oceanography (GNOO, http://www.bo.ingv. it/gnoo/).

Acknowledgements. This work been carried in the framework of the projects MFSTEP, funded by European Commission V Framework Program Energy, Environment and Sustainable Development, and ADRICOSM, funded by Italian Ministry for the Environment and Territory. We thank A. Anav for useful discussion about life and dead of larvae, R. Sciarra for helpful discussion and E. Lombardi and A. Iaccarino for their invaluable support in the management of million of particles.

Edited by: N. Pinardi

\section{References}

Artale, V., Calmanti, S., Pisacane G., and Rupolo, V.: The Atlantic and Mediterranean Sea as connected systems, in: Mediterranean Climate Variability, edited by: Lionello, P., Malanotte-Rizzoli, P., and Boscolo, R., Amsterdam, Elsevier, 283-323, 2006.

Astraldi, M. and Gasparini, G. P.: The seasonal characteristics of the circulation in the North Mediterranean Basin and their relationship with the atmospheric-climatic conditions, J. Geophys. Res., 97, 9531-9540, 1992.

Astraldi, M., Baloupoulos, S., Candela, J., Font, J., Gacic, M., Gasparini, G. P., Manca, B., Theocharis, A., and Tintorè, J.: The role of straits and channel in understanding the characteristics of Mediterranean circulation, Progress. Oceanogr., 44, 65-108, 1999.

Blanke, B. and Raynaud, S.: Kinematics of the Pacific equatorial undercurrent: a Eulerian and Lagrangian approach from GCM results, J. Phys. Oceanogr., 27, 1038-1053, 1997.

Cowen, R. K., Kamazima Lwiza, M. M., Sponaugle, S., Parisa C. B., and Olson, D. B.: Connectivity of Marine Populations: Open or Closed?, Science, 287, 857-859, 2000.

Demirov, E., Pinardi, N., Fratianni, C., Tonani, M., Giacomelli, L., and De mey, P.: Assimilation scheme of Mediterranean Forecasting System: Operational implementation, Ann. Geophys., 21, 189-204, 2003, http://www.ann-geophys.net/21/189/2003/.

Griffa, A., Molcard, A., Raicich, F., and Rupolo, V.: Assessment of the impact of TS assimilation from ARGO floats in The Mediterranean Sea, Ocean Sci., 2, 237-248, 2006, http://www.ocean-sci.net/2/237/2006/.

Guinehut, S., Larnicol, G., and Le Traon, P. Y.: Design of an array of profiling floats in the North Atlantic from model simulations, J. Mar. Syst., 35, 1-9, 2002

Herbaut, C., Mortier, L., and Crépon, M.: A sensitivity study of the general circulation of the western Mediterranean Sea. Part I: The response to density forcing through the straits, J. Phys. Oceanogr., 26, 65-84, 1996.

Herbaut, C., Martel F. L., and Crépon, M.: A sensitivity study of the general circulation of the western Mediterranean Sea. Part II: The response to atmospheric forcing, J. Phys. Oceanogr., 27, 2126-2145, 1997.

Korres, G., Pinardi, N., and Lascaratos, A.: The ocean response to low frequency interannual atmospheric variability in the Mediterranean Sea, J. Climate, 13, 705-731, 2000.
Malanotte-Rizzoli P., Manca, B. B., Ribera d'Alcalà, M., Theocharis, A., Brenner, S., Budillon, G., and Ozsoy, E.,: The Eastern Mediterranean in the 80's and in the 90's: the big transition in the intermediate and deep circulations, Dyn. Atmos. Oceans, 29, 365-395, 1999.

Manzella, G. M. R., Scoccimarro, E., Pinardi, N., and Tonani, M.: Improved near-real time management procedures for the Mediterranean ocean Forecasting System? Volunteer Observing Ships program, Ann. Geophys., 21, 49-62, 2003, http://www.ann-geophys.net/21/49/2003/.

MEDOC group: Observation of Formation of Deep Water in the Mediterranean, Nature, 227, 1037-1040, 1970.

Mertens, C. and Schott, F.: Interannual variability of deep water formation in the Northwestern Mediterranean, J. Phys. Oceanogr., 28, 1410-1424, 1998.

Molcard, A., Griffa, A., and Özgökmen, T.: Lagrangian data assimilation in multilayer primitive equation models, J. Atmos. Oceanic Technol., 22, 70-83, 2005.

Pierini, S. and Rubino, A.: Modeling the Oceanic Circulation in the Area of the Strait of Sicily: The Remotely Forced Dynamics, J. Phys. Oceanogr., 31, 1397-1412, 2001.

Pizzigalli, C., Rupolo, V., Lombardi, E., and Blanke, B.: Seasonal Probability Dispersion Maps in the Mediterranean Sea obtained from the MFS Eulerian velocity fields, J. Geophys. Res., in press, doi:10.1029/2006JC003870, 2007.

Poulain, P.-M., Barbanti, R., Cecco, R., Fayos C., Mauri E., Ursella, L., and Zanasca, P. : Mediterranean Surface Drifter Database: 2 June 1986 to 11 November 1999. Tech. Report 78/2004/OGA/31, OGS, Trieste, Italy (CDROM and http://poseidon.ogs.trieste.it/ drifter/database_med), 2004.

Poulain, P. M.: A profiling float program in the Mediterranean Sea, Argonautics, 6, 2, 2005.

Poulain, P. M., Barbanti, R., Font, J., Cruzado, A., Millot, C., Gertman, I., Griffa, A., Molcard, A., Rupolo, V., Le Bras, S., and Petit de la Villeon, L.: MedARGO: A Drifting Profiler Program in the Mediterranean Sea, Ocean Sci. Discuss, Special Issue "Mediterranean Ocean Forecasting System: toward environmental predictions - the results", 1901-1943, 2006.

Raicich, F.: The assessment of temperature and salinity sampling strategies in the Mediterranean Sea: idealized and real cases, Ocean Sci., 2, 97-112, 2006, http://www.ocean-sci.net/2/97/2006/.

Rinaldi, E.: Studio della circolazione superficiale del Mar Tirreno mediante dati satellitari e Lagrangiani' Tesi di Laurea,' Università La Parthenope, Napoli, 2006.

Robinson, A. R. and Golnaraghi, M.: The physical and dynamical oceanography of the Mediterranean, in: Ocean Processes in Climate Dynamics: Global and Mediterranean Examples, edited by: Malanotte-Rizzoli, P. and Robinson, A. R., Kluwer Academic Publishers, The Netherlands, 255-306, 1994.

Siegel, D. A., Kinlan, B. P., Gaylord, B., and Gaines, S. D.: Lagrangian descriptions of marine larval dispersion, Mar. Ecol. Progr. Ser., 260, 83-96, 2003.

Taillandier, V. and Griffa, A.: Implementation of position assimilation for ARGO floats in a realistic Mediterranean Sea OPA model and twin experiment testing, Ocean Sci., 2, 223-236, 2006, http://www.ocean-sci.net/2/223/2006/. 